\title{
Theoretical aspects of a multiscale analysis of the eigenoscillations of the earth
}

\author{
Volker MICHEL \\ Geomathematics Group \\ Department of Mathematics \\ University of Kaiserslautern \\ P.O. Box 3049 \\ D-67653 Kaiserslautern, Germany \\ michel@mathematik.uni-kl.de \\ Recibido: 16 de Julio de 2002 \\ Aceptado: 21 de Octubre de 2002

\begin{abstract}
The elastic behaviour of the Earth, including its eigenoscillations, is usually described by the Cauchy-Navier equation. Using a standard approach in seismology we apply the Helmholtz decomposition theorem to transform the Fourier transformed Cauchy-Navier equation into two non-coupled Helmholtz equations and then derive sequences of fundamental solutions for this pair of equations using the Mie representation. Those solutions are denoted by the Hansen vectors $L_{n, j}, M_{n, j}$, and $N_{n, j}$ in geophysics. Next we apply the inverse Fourier transform to obtain a function system depending on time and space. Using this basis for the space of eigenoscillations we construct scaling functions and wavelets to obtain a multiresolution for the solution space of the Cauchy-Navier equation.
\end{abstract}

2000 Mathematics Subject Classification: 35J05, 42C40.

Key words: Cauchy-Navier equation, wavelets, multiresolution, Helmholtz equation, Hansen vectors, geomathematics

\section{Introduction}

Wavelet-based multiscale methods have already been applied to a series of geoscientific problems. Whereas the conventional approach of a polynomial expansion is appropriate to cover the global low-scale, i.e. low-degree, trend of the investigated functions, this method reaches its frontiers if local modifications are required, if the available data are not equidistributed, or if huge amounts of data have to be processed for an extremely high resoluting model. In such cases the localizing structures of kernels like wavelets is an essential advantage, since local variations of the data, 
e.g. due to a seismic event, only require a local modification of the model, and the occurring integrals only have to be calculated over a subsection of the domain.

The shape of the Earth and the special data situations made the development of new kinds of wavelets necessary. The first geoscientifically relevant wavelets ([16]) were constructed for a multiscale decomposition of scalar functions on the unit sphere, such as the gravitational potential. Later on, a generalized concept for scalar functions on regular surfaces has been introduced ([14]), allowing a multiscale determination of solutions of (exterior) boundary-value problems. A series of further publications (see e.g. [10], [9]) treated the wavelet analysis of scalar functions on closed surfaces. Relevant applications of those methods are, for instance, the modelling of the gravitational potential, the absolute value of the magnetic field on the Earth's surface or the topography. The obtained scale and detail spaces are usually not orthogonal. Only recently, non-band-limited orthogonal wavelets on the sphere have been discovered ([11]).

However, not every geoscientifical problem can be solved by a pure interpolation or approximation of measured data. Often the acquired data are used to recover quantities, on which those data depend. An example of such an inverse problem is the calculation of the gravitational potential on the Earth's surface from measurements at the satellite orbit. This inversion is discontinuous, such that a regularization is necessary, which has been realized by wavelets ([26], [15]).

The interest in the application of the new multiscale concept to further geoscientifical problems required an extension of the theory and the methods to functions with different domains or different values. The inverse problem of the reconstruction of the Earth's mass density distribution from gravitational measurements needs, for example, the treatment of functions which are defined on the whole Earth and not only the surface. Moreover, on this three-dimensional domain, we may not assume that the functions of interest can be approximated by harmonic polynomials, in contrast to the situation in case of surface problems. In the $3 \mathrm{D}$ case the $\mathrm{L}^{2}$-space can be decomposed into the set of harmonic functions and an orthogonal space of so-called anharmonic functions, where only the harmonic part of the density distribution can be recovered from gravitational data. This particular situation requires two multiscale concepts: one for the regularization of the discontinuous recovery of the harmonic part of the solution ([20], [21], [22], [12]) and one for the determination of the anharmonic part from additional (non-gravitational) data ([12]).

Present satellite missions, such as CHAMP, offer huge amounts of gravitational and magnetic data of the Earth. Since the magnetic field is also available in terms of vector data, the development of spherical vector wavelets ([4], [5]) became necessary. We will show in this paper that a multiscale concept can also be developed for the analysis of the eigenoscillations of the Earth. For this purpose we need vector wavelets for the whole ball. We start with the Fourier transformed Cauchy-Navier equation, which allows, by means of the Helmholtz decomposition, a well-known transformation into an uncoupled pair of Helmholtz equations. For further details on the theory of 
the Cauchy-Navier equation or the Helmholtz equation we refer to e.g. [17], [24], [13], [19], and the references therein.

A standard complete system of solutions represent the so-called Hansen vectors (see e.g. [6] and the references therein; as well as [7] for the cylindrical case). One way to show that this system is a fundamental system is to use the Mie representation, which is a decomposition of solenoidal (i.e. divergence-free in our case) vector fields into toroidal and spheroidal vector fields. The results up to this point are well-known. We will then prove a norm-estimate for the fundamental system in the frequency domain. Then we will apply the inverse Fourier transform to the fundamental solutions to obtain an orthogonal system of functions depending on time and space. Also for this system a norm-estimate is calculated. Finally, we will use this system to develop a multiscale decomposition of the spaces of toroidal and spheroidal eigenoscillations of the Earth. Illustrations of the used kernel functions demonstrate the different levels of time and space localization available by the multiscale approach. This new multiscale approach offers a new way of analysing the interesting phenomenon of the Earth's eigenoscillations. The multiresolution analysis allows the zooming into different spatial/temporal resolutions.

\section{Mathematical Tools}

The space of positive integers (natural numbers) is denoted by $\mathbb{N}$, such that $\mathbb{N}_{0}$ is the space of non-negative integers. The symbol for the space of real numbers is, as usual, $\mathbb{R}$ and $\mathbb{C}$ represents the space of complex numbers. The vector space $\mathbb{C}^{n}, n \in \mathbb{N}$, is equipped with the euclidean scalar product $x \cdot y:=\sum_{i=1}^{n} x_{i} \bar{y}_{i} ; x=\left(x_{1}, \ldots, x_{n}\right)^{\mathrm{T}}, y=$ $\left(y_{1}, \ldots, y_{n}\right)^{\mathrm{T}} \in \mathbb{C}^{n}$; where $\bar{z}$ represents the complex conjugate of $z \in \mathbb{C}$, and the corresponding norm $|x|:=\sqrt{x \cdot x}, x \in \mathbb{C}^{n}$. The vector product in $\mathbb{R}^{3}$ is defined by

$$
x \wedge y:=\left(x_{2} y_{3}-x_{3} y_{2}, x_{3} y_{1}-x_{1} y_{3}, x_{1} y_{2}-x_{2} y_{1}\right)^{\mathrm{T}} ; \quad x, y \in \mathbb{R}^{3} ;
$$

whereas the tensor product $x \otimes y:=\left(x_{i} y_{j}\right)_{i, j=1,2,3} ; x, y \in \mathbb{C}^{3} ;$ yields a second-order tensor $\left(3 \times 3\right.$-matrix). If $A, B \in \mathbb{C}^{3 \times 3}$ are tensors and $x \in \mathbb{C}^{3}$ is a vector, then $A x$ and $A B$ denote the usual multiplications. Moreover, the double-dot product of $A=\left(a_{i j}\right)_{i, j=1,2,3}$ and $B=\left(b_{i j}\right)_{i, j=1,2,3}$ is defined by $A: B:=\sum_{i, j=1}^{3} a_{i j} \overline{b_{i j}} \in \mathbb{C}$.

We will restrict our attention to a spherical model of the Earth denoted by $\overline{S_{\text {int }}}$ which is a ball with centre 0 and radius $\sigma>0$ and is interpreted as the closed inner space of the sphere $S=\left\{x \in \mathbb{R}^{3}:|x|=\sigma\right\}$. Of particular importance for our considerations will also be the unit sphere $\Omega$.

The space $\mathrm{C}^{(k)}\left(D, \mathbb{R}^{n}\right) ; D \subset \mathbb{R}^{m}$ compact, $k \in \mathbb{N}_{0} \cup\{\infty\}, n, m \in \mathbb{N}$; of all $k$-times continuously differentiable functions from $D$ into $\mathbb{R}^{n}$ and, in particular, the space $\mathrm{C}\left(D, \mathbb{R}^{n}\right):=\mathrm{C}^{(0)}\left(D, \mathbb{R}^{n}\right)$ of all continuous functions from $D$ into $\mathbb{R}^{n}$ can be equipped with the maximum norm

$$
\|F\|_{\infty}:=\|F\|_{\mathrm{C}\left(D, \mathbb{R}^{n}\right)}:=\max _{x \in D}|F(x)|, \quad F \in \mathrm{C}\left(D, \mathbb{R}^{n}\right),
$$


where $\left(\mathrm{C}\left(D, \mathbb{R}^{n}\right),\|\cdot\|_{\infty}\right)$ is a Banach space. Moreover, the space $\mathrm{L}^{2}\left(D, \mathbb{C}^{n}\right) ; D \subset \mathbb{R}^{m}$ (Lebesgue) measurable, $n, m \in \mathbb{N}$; of all classes of almost everywhere identical squareintegrable functions from $D$ into $\mathbb{C}^{n}$, equipped with the scalar product

$$
(F, G)_{2}:=(F, G)_{\mathrm{L}^{2}\left(D, \mathbb{C}^{n}\right)}:=\int_{D} F(x) \cdot \overline{G(x)} d x ; \quad F, G \in \mathrm{L}^{2}\left(D, \mathbb{C}^{n}\right)
$$

is a Hilbert space. The corresponding norm is given by $\|F\|_{2}:=\|F\|_{\mathrm{L}^{2}\left(D, \mathbb{C}^{n}\right)}:=$ $\sqrt{(F, F)_{\mathrm{L}^{2}\left(D, \mathbb{C}^{n}\right)}}, F \in \mathrm{L}^{2}\left(D, \mathbb{C}^{n}\right)$. This topology is certainly also valid on $\mathrm{L}^{2}\left(D, \mathbb{R}^{n}\right)$. To simplify notations we will write $\mathrm{C}(D):=\mathrm{C}(D, \mathbb{R}), \mathrm{c}(D):=\mathrm{C}\left(D, \mathbb{R}^{3}\right), \mathrm{L}^{2}(D):=$ $\mathrm{L}^{2}(D, \mathbb{R}), \mathrm{l}^{2}(D):=\mathrm{L}^{2}\left(D, \mathbb{R}^{3}\right)$ etc. for $D \subset \mathbb{R}^{3}$. Analogously, we will denote scalar functions by capital letters and vectorial functions by lower-case letters.

The space $\mathrm{L}^{2}\left(D, \mathbb{C}^{3 \times 3}\right) ; D \subset \mathbb{R}^{m}$ (Lebesgue) measurable, $m \in \mathbb{N}$; of all classes of almost everywhere identical square-integrable functions from $D$ into $\mathbb{C}^{3 \times 3}$, equipped with the scalar product

$$
(\Theta, \Lambda)_{2}:=(\Theta, \Lambda)_{\mathrm{L}^{2}\left(D, \mathbb{C}^{3 \times 3}\right)}:=\int_{D} \Theta(x): \overline{\Lambda(x)} d x ; \quad \Theta, \Lambda \in \mathrm{L}^{2}\left(D, \mathbb{C}^{3 \times 3}\right) ;
$$

is a Hilbert space. The corresponding norm is given by $\|\Theta\|_{2}:=\|\Theta\|_{\mathrm{L}^{2}\left(D, \mathbb{C}^{3 \times 3}\right)}:=$ $\sqrt{(\Theta, \Theta)_{\mathrm{L}^{2}\left(D, \mathbb{C}^{3 \times 3}\right)}}, \Theta \in \mathrm{L}^{2}\left(D, \mathbb{C}^{3 \times 3}\right)$.

The Earth model $\overline{S_{\text {int }}}$ motivates a separation ansatz $\left.\left.u(r \xi)=G(r) y(\xi) ; r \in\right] 0, \sigma\right], \xi \in$ $\Omega$; such that a more detailed treatment of functions on the unit sphere $\Omega$ is required for our investigations. An intensive discussion of scalar, vectorial and tensorial functions on the sphere and their approximations can be found in [10], such that we merely try to give a concise overview of the most important tools here.

The gradient grad $=\nabla$ and the Laplace operator $\Delta$, defined by $\Delta_{x}:=\sum_{i=1}^{3} \frac{\partial^{2}}{\partial x_{i}^{2}}$, can be separated into a radial and an angular part in the following way:

$$
\begin{aligned}
\nabla_{r \xi} F(r \xi) & =\xi \frac{\partial}{\partial r} F(r \xi)+\frac{1}{r} \nabla_{\xi}^{*} F(r \xi) \\
\Delta_{r \xi} G(r \xi) & =\frac{\partial^{2}}{\partial r^{2}} G(r \xi)+\frac{2}{r} \frac{\partial}{\partial r} G(r \xi)+\frac{1}{r^{2}} \Delta_{\xi}^{*} G(r \xi)
\end{aligned}
$$

$r \in \mathbb{R}^{+}, \xi \in \Omega, F \in \mathrm{C}^{(1)}(D), G \in \mathrm{C}^{(2)}(D), r \xi \in D \subset \mathbb{R}^{3}$. The spherical parts $\nabla^{*}$ and $\Delta^{*}$ are called surface gradient and Beltrami operator, respectively. Their representation in spherical coordinates can easily be derived as well as the representation of the surface curl gradient $L^{*}$ defined by the vector product

$$
L_{\xi}^{*} F(r \xi):=\xi \wedge \nabla_{\xi}^{*} F(r \xi) ; \quad r \in \mathbb{R}^{+}, \xi \in \Omega, F \in \mathrm{C}^{(1)}(D), r \xi \in D \subset \mathbb{R}^{3} .
$$


The divergence and the curl of a vector field $f \in \mathrm{c}^{(1)}(D), D \subset \mathbb{R}^{3}$, are defined by

$$
\begin{aligned}
\operatorname{div} f:=\nabla \cdot f & :=\sum_{j=1}^{3} \frac{\partial F_{j}}{\partial x_{j}}, \\
\operatorname{curl} f:=\nabla \wedge f & :=\left(\frac{\partial F_{3}}{\partial x_{2}}-\frac{\partial F_{2}}{\partial x_{3}}, \frac{\partial F_{1}}{\partial x_{3}}-\frac{\partial F_{3}}{\partial x_{1}}, \frac{\partial F_{2}}{\partial x_{1}}-\frac{\partial F_{1}}{\partial x_{2}}\right)^{\mathrm{T}},
\end{aligned}
$$

where $f=\left(F_{1}, F_{2}, F_{3}\right)^{\mathrm{T}}$. Note that

$$
\operatorname{curl}_{x}(x F(x))=F(x) \operatorname{curl}_{x} x-x \wedge \nabla_{x} F(x)=-L_{\xi}^{*} F(r \xi)
$$

for $F \in \mathrm{C}^{(1)}(D), x=r \xi \in D \subset \mathbb{R}^{3}$.

An important role in the theory of functions on the sphere plays the space $\operatorname{Harm}_{n}(\Omega)$ of spherical harmonics, i.e. homogeneous harmonic polynomials of degree $n \in \mathbb{N}_{0}$ restricted to the unit sphere $\Omega$. This space has the dimension $2 n+1$ such that we are able to assume that a complete $\mathrm{L}^{2}(\Omega)$-orthonormal system $\left\{Y_{n, j}\right\}_{j=1, \ldots, 2 n+1}$ in $\operatorname{Harm}_{n}(\Omega)$ is given. Whenever we write $Y$ with a double-index we refer to such a complete orthonormal system. Since two spherical harmonics $Y_{n} \in \operatorname{Harm}_{n}(\Omega), Y_{m} \in$ $\operatorname{Harm}_{m}(\Omega)$ of different degrees $n \neq m$ are always orthogonal, i.e. $\left(Y_{n}, Y_{m}\right)_{\mathrm{L}^{2}(\Omega)}=0$, the whole system $\left\{Y_{n, j}\right\}_{n \in \mathbb{N}_{0} ; j=1, \ldots, 2 n+1}$ is automatically an orthonormal system in $\mathrm{L}^{2}(\Omega)$. Moreover, one can prove that such a system is also complete, such that every function $F \in \mathrm{L}^{2}(\Omega)$ satisfies

$$
\lim _{N \rightarrow \infty}\left\|F-\sum_{n=0}^{N} \sum_{j=1}^{2 n+1}\left(F, Y_{n, j}\right)_{\mathrm{L}^{2}(\Omega)} Y_{n, j}\right\|_{\mathrm{L}^{2}(\Omega)}=0 .
$$

We will briefly write " $F=\sum_{n=0}^{\infty} \sum_{j=1}^{2 n+1}\left(F, Y_{n, j}\right)_{\mathrm{L}^{2}(\Omega)} Y_{n, j}$ in the sense of $\mathrm{L}^{2}(\Omega)$ “.

Furthermore, the spherical harmonics have the exposed property to be the only on $\Omega$ infinitely often differentiable eigenfunctions of the Beltrami operator. We have

$$
\Delta^{*} Y_{n}=-n(n+1) Y_{n}
$$

for all $Y_{n} \in \operatorname{Harm}_{n}(\Omega)$ and all $n \in \mathbb{N}_{0}$.

Using a scalar system $\left\{Y_{n, j}\right\}_{n \in \mathbb{N}_{0} ; j=1, \ldots, 2 n+1}$ we obtain a complete orthonormal vectorial system $\left\{y_{n, j}^{(i)}\right\}_{i=1,2,3 ; n \geq 0_{i} ; j=1, \ldots, 2 n+1}$ in $\mathrm{l}^{2}(\Omega)$, given by

$$
y_{n, j}^{(i)}:=\left(\mu_{n}^{(i)}\right)^{-1 / 2} o^{(i)} Y_{n, j} ; j=1, \ldots, 2 n+1 ; n \geq 0_{i}:=\left\{\begin{array}{ll}
0, & \text { if } i=1 \\
1, & \text { if } i \in\{2 ; 3\}
\end{array},\right.
$$

where the operators $o^{(i)} ; i=1,2,3$; are defined by

$$
o_{\xi}^{(1)} F(\xi)=\xi F(\xi), \quad \xi \in \Omega, F \in \mathrm{C}(\Omega),
$$




$$
\begin{aligned}
& o_{\xi}^{(2)} F(\xi)=\nabla_{\xi}^{*} F(\xi), \quad \xi \in \Omega, F \in \mathrm{C}^{(1)}(\Omega), \\
& o_{\xi}^{(3)} F(\xi)=L_{\xi}^{*} F(\xi), \quad \xi \in \Omega, F \in \mathrm{C}^{(1)}(\Omega)
\end{aligned}
$$

and the normalizing constants $\left(\mu_{n}^{(i)}\right)^{-1 / 2}, i=1,2,3, n \geq 0_{i}$, are given by

$$
\mu_{n}^{(i)}:=\left\{\begin{array}{cl}
1, & \text { if } i=1 \\
n(n+1), & \text { if } i \in\{2 ; 3\}
\end{array} .\right.
$$

It is easy to check that vector spherical harmonics $o^{(i)} Y_{n}, Y_{n} \in \operatorname{Harm}_{n}(\Omega), n \geq 0_{i}$, $i \in\{1,2,3\}$, satisfy

$$
\begin{array}{lll}
\xi \wedge\left(o^{(1)} Y_{n}\right)(\xi)=0, & \xi \cdot\left(o^{(2)} Y_{n}\right)(\xi)=0, & \xi \cdot\left(o^{(3)} Y_{n}\right)(\xi)=0, \\
L_{\xi}^{*} \cdot\left(o^{(2)} Y_{n}\right)(\xi)=0, & \nabla_{\xi}^{*} \cdot\left(o^{(3)} Y_{n}\right)(\xi)=0
\end{array}
$$

for all $\xi \in \Omega$. Not only are the functions $y_{n, j}^{(i)}$ orthonormal in the $\mathrm{l}^{2}(\Omega)$-sense, we also have the euclidean orthogonality in the following sense:

$$
\left(o_{\xi}^{(i)} F(\xi)\right) \cdot\left(o_{\xi}^{(j)} F(\xi)\right)=0 \text { if } i \neq j
$$

for $F \in \mathrm{C}^{(1)}(\Omega), \xi \in \Omega$, and $i, j \in\{1,2,3\}$.

The application of the Laplace operator $\Delta$ and the Beltrami operator $\Delta^{*}$ to vectorial functions is always meant componentwise. The combination of the Beltrami operator with the $o^{(i)}$-operators yields the following identities:

$$
\begin{aligned}
& \Delta^{*} o^{(1)} F=o^{(1)}\left(\Delta^{*}-2\right) F+2 o^{(2)} F, \quad F \in \mathrm{C}^{(2)}(\Omega), \\
& \Delta^{*} o^{(2)} F=-2 o^{(1)} \Delta^{*} F+o^{(2)} \Delta^{*} F, \quad F \in \mathrm{C}^{(3)}(\Omega), \\
& \Delta^{*} o^{(3)} F=o^{(3)} \Delta^{*} F, \quad F \in \mathrm{C}^{(3)}(\Omega) .
\end{aligned}
$$

\section{From the Cauchy-Navier equation to the Helmholtz equation}

The Fourier-transformed Cauchy-Navier equation for a homogeneous medium is represented by

$$
\alpha^{2} \operatorname{grad} \operatorname{div} u-\beta^{2} \operatorname{curl} \operatorname{curl} u+\omega^{2} u=0,
$$

if the body forces are neglected. The quantities $\alpha, \beta \in \mathbb{R}^{+}$, which are in the homogeneous case assumed to be constant, are the speeds of compressional and shear body waves, respectively, where $\alpha>\beta$. The unknown quantity $u(\cdot, \omega) \in \mathrm{c}^{(2)}\left(\overline{S_{\text {int }}}\right)$ is a small displacement depending on the angular frequency $\omega \in \mathbb{R} \backslash\{0\}$. Note that, for mathematical reasons, we also allow $\omega<0$. For further details on the derivation of this equation we refer to e.g. [6].

Following an approach, e.g. described in [6] and [17], we will now show that (1) is valid if two non-coupled Helmholtz equations are satisfied. We will further show that 
the opposite conclusion is easy to verify.

The Helmholtz decomposition theorem (see e.g. [23]) says that for every continuously differentiable vector function $u: \overline{S_{\text {int }}} \rightarrow \mathbb{R}^{3}$ there exist continuously differentiable vector functions $u_{\alpha}, u_{\beta}$ on $\overline{S_{\text {int }}}$ such that $u=u_{\alpha}+u_{\beta}$ and $\operatorname{curl} u_{\alpha}=0$ as well as $\operatorname{div} u_{\beta}=0$. Hence, we find

$$
\alpha^{2} \operatorname{grad} \operatorname{div} u_{\alpha}-\beta^{2} \operatorname{curl} \operatorname{curl} u_{\beta}+\omega^{2}\left(u_{\alpha}+u_{\beta}\right)=0 .
$$

Since $\Delta=\operatorname{grad}$ div - curl curl this is equivalent to

$$
\alpha^{2}\left(\Delta u_{\alpha}+\text { curl curl } u_{\alpha}\right)+\beta^{2}\left(\Delta u_{\beta}-\operatorname{grad} \operatorname{div} u_{\beta}\right)+\omega^{2}\left(u_{\alpha}+u_{\beta}\right)=0,
$$

which is true if

$$
\left(\Delta+\frac{\omega^{2}}{\alpha^{2}}\right) u_{\alpha}=0
$$

and

$$
\left(\Delta+\frac{\omega^{2}}{\beta^{2}}\right) u_{\beta}=0 .
$$

An equation of the type (3) or (4) is called Helmholtz equation.

Vice versa, it suffices to consider only such pairs $\left(u_{\alpha}, u_{\beta}\right)$ solving (3) and (4). If in general

$$
\left(\Delta+\frac{\omega^{2}}{\alpha^{2}}\right) u_{\alpha}=f, \quad\left(\Delta+\frac{\omega^{2}}{\beta^{2}}\right) u_{\beta}=g,
$$

then (2) implies $f=-\frac{\beta^{2}}{\alpha^{2}} g$. Using Schwarz's Theorem (and assuming $f \in \mathrm{c}^{(1)}\left(\overline{S_{\text {int }}}\right)$ ) we find

$$
\operatorname{curl} f=\Delta\left(\operatorname{curl} u_{\alpha}\right)+\frac{\omega^{2}}{\alpha^{2}} \operatorname{curl} u_{\alpha}=0,
$$

such that $f$ is a gradient field $f=\nabla F$, and

$$
\operatorname{div} f=-\frac{\beta^{2}}{\alpha^{2}} \Delta \operatorname{div} u_{\beta}-\frac{\omega^{2}}{\alpha^{2}} \operatorname{div} u_{\beta}=0,
$$

such that $F$ is harmonic,

$$
\Delta F=\operatorname{div} \operatorname{grad} F=\operatorname{div} f=0,
$$

and, therefore, also $f$ :

$$
\Delta f=\Delta \operatorname{grad} F=\operatorname{grad} \Delta F=0 .
$$

However, this implies that

$$
\begin{aligned}
& \Delta\left(u_{\alpha}-\frac{\alpha^{2}}{\omega^{2}} f\right)+\frac{\omega^{2}}{\alpha^{2}}\left(u_{\alpha}-\frac{\alpha^{2}}{\omega^{2}} f\right)=0, \\
& \Delta\left(u_{\beta}+\frac{\alpha^{2}}{\omega^{2}} f\right)+\frac{\omega^{2}}{\beta^{2}}\left(u_{\beta}+\frac{\alpha^{2}}{\omega^{2}} f\right)=0 .
\end{aligned}
$$


Since $\left(u_{\alpha}-\frac{\alpha^{2}}{\omega^{2}} f\right)+\left(u_{\beta}+\frac{\alpha^{2}}{\omega^{2}} f\right)=u_{\alpha}+u_{\beta}$, we do not obtain any new solution if we also take into account inhomogeneous Helmholtz equations (with continuously differentiable right hand sides). Thus, it suffices to investigate the solutions of (3) and (4).

\section{Fundamental Solutions}

For the derivation of a system of fundamental solutions we use a decomposition theorem called the Mie representation (see e.g. [1], [2], [3]).

Theorem 4.1. Let $f \in \mathrm{c}^{(1)}\left(\overline{S_{\mathrm{int}}}\right)$ be a solenoidal vector field, i.e. $\operatorname{div} f=0$. Then there exist unique vector fields $p \in \mathrm{c}^{(1)}\left(\overline{S_{\mathrm{int}}}\right)$ and $q \in \mathrm{c}^{(1)}\left(\overline{S_{\mathrm{int}}}\right)$ satisfying

$$
f=p+q
$$

such that $p$ is poloidal and $q$ is toroidal, i.e. there exist scalar functions $P \in \mathrm{C}^{(3)}\left(\overline{S_{\mathrm{int}}}\right)$ and $Q \in \mathrm{C}^{(2)}\left(\overline{S_{\text {int }}}\right)$ such that

$$
p=\nabla \wedge L^{*} P, \quad q=L^{*} Q
$$

where the scalars $P$ and $Q$ are uniquely determined up to an added function that only depends on the euclidean norm of the argument.

In [6] a system of so-called Hansen vectors is given that consists of solutions of the Helmholtz equations (3) and (4). An argumentation for the completeness is given. We will now give an alternative proof that every solution can be represented as a linear combination of Hansen vectors.

Theorem 4.2. The general $\mathrm{C}^{(\infty)}\left(\overline{S_{\text {int }}}\right)$-solution of $(3)$ is given by

$$
u_{\alpha}=\nabla \varphi_{\alpha}, \quad \Delta \varphi_{\alpha}+\frac{\omega^{2}}{\alpha^{2}} \varphi_{\alpha}=0
$$

and the general $\mathrm{C}^{(\infty)}\left(\overline{S_{\mathrm{int}}}\right)$-solution of $(4)$ is given by

$$
u_{\beta}=u_{\beta, 1}+u_{\beta, 2}
$$

with

$$
\begin{gathered}
u_{\beta, 1}=L^{*} \varphi_{\beta, 1}, \quad u_{\beta, 2}=\nabla \wedge L^{*} \varphi_{\beta, 2}, \\
\Delta \varphi_{\beta, j}+\frac{\omega^{2}}{\beta^{2}} \varphi_{\beta, j}=0, \quad j \in\{1,2\} .
\end{gathered}
$$

Proof. Note that it is already well-known that every $\mathrm{C}^{(2)}$-solution of the Helmholtz equation is automatically a $\mathrm{C}^{(\infty)}$-solution (see e.g. [24]). The condition $\nabla \wedge u_{\alpha}=0$ 
on the starshaped domain $\overline{S_{\text {int }}}$ implies the existence of a potential $\tilde{\varphi}_{\alpha}$ such that $u_{\alpha}=\nabla \tilde{\varphi}_{\alpha}$. Hence,

$$
\Delta\left(\nabla \tilde{\varphi}_{\alpha}\right)+\frac{\omega^{2}}{\alpha^{2}} \nabla \tilde{\varphi}_{\alpha}=0 \Leftrightarrow \nabla\left(\Delta \tilde{\varphi}_{\alpha}+\frac{\omega^{2}}{\alpha^{2}} \tilde{\varphi}_{\alpha}\right)=0 .
$$

This is true if and only if $\Delta \tilde{\varphi}_{\alpha}+\frac{\omega^{2}}{\alpha^{2}} \tilde{\varphi}_{\alpha}$ is constant. If we denote this constant by $\gamma \frac{\omega^{2}}{\alpha^{2}}, \gamma \in \mathbb{R}$, and define $\varphi_{\alpha}:=\tilde{\varphi}_{\alpha}-\gamma$, we obtain that (7) is equivalent to

$$
\Delta \varphi_{\alpha}+\frac{\omega^{2}}{\alpha^{2}} \varphi_{\alpha}=0
$$

The solution $u_{\beta}$ of (4) is solenoidal due to the Helmholtz decomposition. Thus, there exist scalars $P, Q$ with

$$
u_{\beta}=\nabla \wedge L^{*} P+L^{*} Q .
$$

With elementary calculations one can easily verify that $\Delta L^{*}=L^{*} \Delta$ and $\Delta$ curl $=$ $\operatorname{curl} \Delta$, such that (4) is equivalent to

$$
\operatorname{curl} L^{*}\left(\Delta P+\frac{\omega^{2}}{\beta^{2}} P\right)+L^{*}\left(\Delta Q+\frac{\omega^{2}}{\beta^{2}} Q\right)=0 .
$$

This is a Mie representation of the zero function. Hence, the scalars $\tilde{P}:=\Delta P+\frac{\omega^{2}}{\beta^{2}} P$ and $\tilde{Q}:=\Delta Q+\frac{\omega^{2}}{\beta^{2}} Q$ are merely radially dependent. Moreover, we know that the poloidal part and the toroidal part are unique such that we may conclude that $L^{*} \tilde{Q}=$ 0 . Now let $\hat{Q}$ be an arbitrary scalar such that

$$
L^{*}\left(\Delta \hat{Q}+\frac{\omega^{2}}{\beta^{2}} \hat{Q}\right)=0 .
$$

Thus, there exists a function $G$ on $[0, \sigma]$ such that

$$
\Delta_{x} \hat{Q}(x)+\frac{\omega^{2}}{\beta^{2}} \hat{Q}(x)=G(|x|), \quad x \in \overline{S_{\mathrm{int}}} .
$$

According to [27], p. 588, the function $V$, given by

$$
V(x)=\frac{1}{4 \pi} \int_{\frac{S_{\text {int }}}{}} \frac{e^{-i k|x-y|}}{|x-y|} G(|y|) d y, \quad k=\frac{\omega^{2}}{\beta^{2}},
$$

satisfies the inhomogeneous Helmholtz equation (8). Moreover, with $x=|x| \xi, y=r \eta$ we obtain

$$
\begin{aligned}
V(x) & =\frac{1}{4 \pi} \int_{0}^{\sigma} r^{2} G(r) \int_{\Omega} \frac{e^{-i k \sqrt{|x|^{2}+r^{2}-2|x| r \xi \cdot \eta}}}{\sqrt{|x|^{2}+r^{2}-2|x| r \xi \cdot \eta}} d \omega(\eta) d r \\
& =\frac{1}{4 \pi} \int_{0}^{\sigma} r^{2} G(r) 2 \pi \int_{-1}^{1} \frac{e^{-i k \sqrt{|x|^{2}+r^{2}-2|x| r t}}}{\sqrt{|x|^{2}+r^{2}-2|x| r t}} d t d r,
\end{aligned}
$$


i.e. $V(x)$ only depends on $|x|$. Consequently, $Q$ given by $Q=\hat{Q}-V$ satisfies

$$
\Delta Q+\frac{\omega^{2}}{\beta^{2}} Q=0
$$

and

$$
L^{*} Q=L^{*}(\hat{Q}-V)=L^{*} \hat{Q}-L^{*} V=L^{*} \hat{Q},
$$

such that it suffices to use the solutions of the homogeneous Helmholtz equation. In other words, every toroidal part $u_{\beta, 1}$ of $u_{\beta}$ may be represented by

$$
u_{\beta, 1}=L^{*} Q, \quad \Delta Q+\frac{\omega^{2}}{\beta^{2}} Q=0 .
$$

Finally, let $\hat{P}$ be an arbitrary scalar such that $\nabla \wedge L^{*}\left(\Delta \hat{P}+\frac{\omega^{2}}{\beta^{2}} \hat{P}\right)=0$. Then there exists a function $F$ on $[0, \sigma]$ with

$$
\Delta_{x} \hat{P}(x)+\frac{\omega^{2}}{\beta^{2}} \hat{P}(x)=F(|x|), \quad x \in \overline{S_{\text {int }}} .
$$

In analogy to above Equation (9) has a merely radially dependent solution $V$ such that $P:=\hat{P}-V$ satisfies

$$
\nabla \wedge L^{*} P=\nabla \wedge L^{*} \hat{P}, \quad \Delta P+\frac{\omega^{2}}{\beta^{2}} P=0 .
$$

In seismology one finds the notations $L:=u_{\alpha}, M:=u_{\beta, 1}$ and $N:=u_{\beta, 2}$ for the Hansen vectors (see e.g. [6]). Since we use lower-case letters for vectorial functions our notations will differ from the seismological ones. However, to avoid conflicts with enumeration indices we utilize Gothic type letters $\mathfrak{l}, \mathfrak{m}$, and $\mathfrak{n}$.

Lemma 4.3. The Hansen vectors have the following properties ([6]):

$$
\begin{gathered}
\operatorname{div} \mathfrak{m}=0, \quad x \cdot \mathfrak{m}=0, \\
x \cdot \operatorname{curl} \mathfrak{n}=x \cdot \operatorname{curl} \mathfrak{l}=0 .
\end{gathered}
$$

The lemma can be derived from the representations (5) and (6) with elementary calculations. Note that solutions of type $\mathfrak{l}$ or $\mathfrak{n}$ (or linear combinations of them) are called spheroidal oscillations ([6]).

We will now apply a separation ansatz $\varphi(r \xi)=F(r) Y(\xi), r \in] 0, \sigma], \xi \in \Omega$, to the potentials of the Hansen vectors (cf. [6]).

Theorem 4.4. The separation ansatz

$$
\varphi_{\alpha}(r \xi)=G(r) \tilde{Y}(\xi), \quad \varphi_{\beta, 1}(r \xi)=F_{1}(r) Y_{1}(\xi), \quad \varphi_{\beta, 2}(r \xi)=F_{2}(r) Y_{2}(\xi),
$$


$r \in] 0, \sigma], \xi \in \Omega$, implies the following form of the Hansen vectors:

$$
\begin{aligned}
& \mathfrak{l}(r \xi)=G^{\prime}(r) o_{\xi}^{(1)} \tilde{Y}(\xi)+r^{-1} G(r) o_{\xi}^{(2)} \tilde{Y}(\xi), \\
& \mathfrak{m}(r \xi)=F_{1}(r) o_{\xi}^{(3)} Y_{1}(\xi), \\
& \mathfrak{n}(r \xi)=-\left(r F_{2}^{\prime \prime}(r)+2 F_{2}^{\prime}(r)+\frac{\omega^{2}}{\beta^{2}} r F_{2}(r)\right) o_{\xi}^{(1)} Y_{2}(\xi)-\left(r^{-1} F_{2}(r)+F_{2}^{\prime}(r)\right) o_{\xi}^{(2)} Y_{2}(\xi), \\
&r \in] 0, \sigma], \xi \in \Omega .
\end{aligned}
$$

Proof. Let $r \in] 0, \sigma]$ and $\xi \in \Omega$. Since $\mathfrak{l}=\nabla \varphi_{\alpha}$ we find

$$
\mathfrak{l}(r \xi)=\left(\xi \frac{\partial}{\partial r}+\frac{1}{r} \nabla_{\xi}^{*}\right) G(r) \tilde{Y}(\xi)=G^{\prime}(r) o_{\xi}^{(1)} \tilde{Y}(\xi)+r^{-1} G(r) o_{\xi}^{(2)} \tilde{Y}(\xi) .
$$

For $\mathfrak{m}=L^{*} \varphi_{\beta, 1}, x=r \xi$ we obtain

$$
\mathfrak{m}(r \xi)=\xi \wedge \nabla_{\xi}^{*}\left(F_{1}(r) Y_{1}(\xi)\right)=F_{1}(r) o_{\xi}^{(3)} Y_{1}(\xi) .
$$

Finally, $\mathfrak{n}=-\operatorname{curl} \operatorname{curl}\left(x \varphi_{\beta, 2}\right)=-\operatorname{grad} \operatorname{div}\left(x \varphi_{\beta, 2}\right)+\Delta\left(x \varphi_{\beta, 2}\right)$ can be manipulated in the following way $(x=r \xi)$ :

$$
\begin{aligned}
\mathfrak{n}(r \xi)=-\operatorname{grad}_{x} \operatorname{div}_{x}\left(x \varphi_{\beta, 2}(x)\right)+2 \nabla_{x} \varphi_{\beta, 2}(x)+\left(\Delta_{x} \varphi_{\beta, 2}(x)\right) x \\
=-\nabla_{x} \sum_{j=1}^{3} \frac{\partial\left(x_{j} \varphi_{\beta, 2}(x)\right)}{\partial x_{j}}+2\left(\xi F_{2}^{\prime}(r) Y_{2}(\xi)+r^{-1} \nabla_{\xi}^{*} Y_{2}(\xi) F_{2}(r)\right)-\frac{\omega^{2}}{\beta^{2}} F_{2}(r) Y_{2}(\xi) r \xi \\
=-\nabla_{x}\left(3 \varphi_{\beta, 2}(x)+x \cdot \nabla_{x} \varphi_{\beta, 2}(x)\right)-\left(\frac{\omega^{2}}{\beta^{2}} r F_{2}(r)-2 F_{2}^{\prime}(r)\right) \xi Y_{2}(\xi)+\frac{2}{r} F_{2}(r) \nabla_{\xi}^{*} Y_{2}(\xi) \\
=-4\left(\xi F_{2}^{\prime}(r) Y_{2}(\xi)+r^{-1} F_{2}(r) o_{\xi}^{(2)} Y_{2}(\xi)\right)-\left(\left(\nabla_{x} \otimes \nabla_{x}\right) \varphi_{\beta, 2}(x)\right) x \\
\quad-\left(\frac{\omega^{2}}{\beta^{2}} r F_{2}(r)-2 F_{2}^{\prime}(r)\right) o_{\xi}^{(1)} Y_{2}(\xi)+2 r^{-1} F_{2}(r) o_{\xi}^{(2)} Y_{2}(\xi) .
\end{aligned}
$$

Further elementary calculations show that the Hesse tensor of $\varphi_{\beta, 2}$ (see also [10], pp. 379 for the representation of the Hesse tensor in the $(r, \xi)$-coordinate system) satisfies

$$
\left(\left(\nabla_{x} \otimes \nabla_{x}\right) \varphi_{\beta, 2}(x)\right) \xi=F_{2}^{\prime \prime}(r) o_{\xi}^{(1)} Y_{2}(\xi)+r^{-1}\left(F_{2}^{\prime}(r)-r^{-1} F_{2}(r)\right) o_{\xi}^{(2)} Y_{2}(\xi)
$$

such that

$\mathfrak{n}(r \xi)=-\left(r F_{2}^{\prime \prime}(r)+2 F_{2}^{\prime}(r)+\frac{\omega^{2}}{\beta^{2}} r F_{2}(r)\right) o_{\xi}^{(1)} Y_{2}(\xi)-\left(r^{-1} F_{2}(r)+F_{2}^{\prime}(r)\right) o_{\xi}^{(2)} Y_{2}(\xi)$

Using Theorem 4.2, which says that the potentials $\varphi(r \xi)=F(r) Y(\xi)$ may be regarded as solutions of scalar Helmholtz equations

$$
\Delta \varphi+\frac{\omega^{2}}{\gamma^{2}} \varphi=0
$$


$\gamma=\alpha$ or $\gamma=\beta$, the separation ansatz yields (see also [6])

$$
F^{\prime \prime}(r) Y(\xi)+\frac{2}{r} F^{\prime}(r) Y(\xi)+\frac{1}{r^{2}} F(r) \Delta_{\xi}^{*} Y(\xi)+\frac{\omega^{2}}{\gamma^{2}} F(r) Y(\xi)=0,
$$

$r \in] 0, \sigma], \xi \in \Omega$. Since $\left\{Y_{n, j}\right\}_{n \in \mathbb{N}_{0}, j \in\{1, \ldots, 2 n+1\}}$ represents a complete orthonormal system in $\mathrm{L}^{2}(\Omega)$, we may restrict our construction of a fundamental system to functions with spherical part $Y=Y_{n, j}$. In this case we see that (10) is equivalent to

$$
\left.\left.F^{\prime \prime}(r)+\frac{2}{r} F^{\prime}(r)+\left(\frac{\omega^{2}}{\gamma^{2}}-\frac{n(n+1)}{r^{2}}\right) F(r)=0, \quad r \in\right] 0, \sigma\right] .
$$

Note that the substitution $F(r)=r^{-1 / 2} K\left(\frac{\omega}{\gamma} r\right), s:=\frac{\omega}{\gamma} r$ in (11) yields the Bessel differential equation

$$
s^{2} K^{\prime \prime}(s)+s K^{\prime}(s)+\left(s^{2}-(n+1 / 2)^{2}\right) K(s)=0, \quad n \in \mathbb{N}_{0},
$$

where the Bessel functions $J_{n+1 / 2}$ and $J_{-n-1 / 2}$ represent a fundamental system of (12) (cf. e.g. [6]). Note that $\left|J_{n+1 / 2}(x)\right|=\left|J_{n+1 / 2}(-x)\right|,\left|J_{-n-1 / 2}(x)\right|=\left|J_{-n-1 / 2}(-x)\right|$ for every $n \in \mathbb{N}_{0}$ and every $x \in \mathbb{R}$ ([30], p. 201; [31], p. 622). In [28], p. 167 we find that $J_{\alpha}(z) \sim z^{\alpha}$ as $z \rightarrow 0+$ for all $\alpha \in \mathbb{R}$ with $-\alpha \notin \mathbb{N}_{0}$. Since we are interested in continuous functions, more precisely in $\mathrm{C}^{(\infty)}\left(\overline{S_{\text {int }}}\right)$-functions, only $J_{n+1 / 2}$ comes into question.

Definition 4.5. The function $\mathfrak{J}_{n}: \mathbb{R} \backslash\{0\} \rightarrow \mathbb{C}, n \in \mathbb{N}_{0}$, given by

$$
\mathfrak{J}_{n}(s):=\left(\frac{\pi}{2 s}\right)^{1 / 2} J_{n+1 / 2}(s), \quad s \in \mathbb{R} \backslash\{0\},
$$

is called $n$-th spherical Bessel function.

Note that common symbols for spherical Bessel functions are, for instance, $j_{n}$ (see e.g. [6]) and $\psi_{n}$ (see e.g. [18]). However, this does not fit to our convention on the utilization of capital letters for scalar functions.

We now find a well-known fundamental system (cf. [6]) for the solution space of the Cauchy-Navier equation (1). Remember that $\omega$ is also a variable of the oscillations which has been regarded as arbitrary but fixed up to this point but should now also be taken into account. Note that $\omega^{1 / 2} \in \mathbb{C} \backslash \mathbb{R}$ if $\omega<0$.

For the representation of the fundamental system the relations (see e.g. [6]; [30], p. 45)

$$
\begin{aligned}
\frac{2 n+1}{s} \mathfrak{J}_{n}(s) & =\mathfrak{J}_{n-1}(s)+\mathfrak{J}_{n+1}(s), \\
\frac{d}{d s} \mathfrak{J}_{n}(s) & =\frac{n}{2 n+1} \mathfrak{J}_{n-1}(s)-\frac{n+1}{2 n+1} \mathfrak{J}_{n+1}(s),
\end{aligned}
$$


which are valid for $n \in \mathbb{Z}, s \in \mathbb{R} \backslash\{0\}$, are used, such that we have

$$
\frac{1}{r} \mathfrak{J}_{n}\left(\frac{\omega}{\beta} r\right)+\frac{\omega}{\beta} \mathfrak{J}_{n}^{\prime}\left(\frac{\omega}{\beta} r\right)=\frac{\omega}{\beta}\left(\frac{n+1}{2 n+1} \mathfrak{J}_{n-1}\left(\frac{\omega}{\beta} r\right)-\frac{n}{2 n+1} \mathfrak{J}_{n+1}\left(\frac{\omega}{\beta} r\right)\right) .
$$

Moreover, since $\mathfrak{J}_{n}$ is a solution of (11) it satisfies

$$
r\left(\frac{d^{2}}{d r^{2}}+\frac{2}{r} \frac{d}{d r}\right) \mathfrak{J}_{n}\left(\frac{\omega}{\gamma} r\right)=\left(\frac{n(n+1)}{r}-\frac{\omega^{2}}{\gamma^{2}} r\right) \mathfrak{J}_{n}\left(\frac{\omega}{\gamma} r\right) .
$$

Finally, we use the fact, that $\frac{\gamma}{\omega} \mathfrak{J}_{n}$ certainly also solves (11), except in the negligible case $\omega=0$.

Theorem 4.6. A fundamental system of the Cauchy-Navier equation (1) is given by

$$
\begin{aligned}
\mathfrak{l}_{n, j}(\omega, r \xi):= & \mathfrak{J}_{n}^{\prime}\left(\frac{\omega}{\alpha} r\right) y_{n, j}^{(1)}(\xi)+\frac{\alpha}{\omega r} \mathfrak{J}_{n}\left(\frac{\omega}{\alpha} r\right) \sqrt{n(n+1)} y_{n, j}^{(2)}(\xi) ; \\
& n \in \mathbb{N}_{0}, j \in\{1, \ldots, 2 n+1\} ; \\
\mathfrak{m}_{n, j}(\omega, r \xi):= & \mathfrak{J}_{n}\left(\frac{\omega}{\beta} r\right) y_{n, j}^{(3)}(\xi) ; \quad n \in \mathbb{N}, j \in\{1, \ldots, 2 n+1\} ; \\
\mathfrak{n}_{n, j}(\omega, r \xi):= & \frac{\beta}{\omega}\left(\frac{1}{r}+\frac{d}{d r}\right) \mathfrak{J}_{n}\left(\frac{\omega}{\beta} r\right) \sqrt{n(n+1)} y_{n, j}^{(2)}(\xi) \\
& +\frac{\beta}{\omega}\left(r \frac{d^{2}}{d r^{2}}+2 \frac{d}{d r}+\frac{\omega^{2}}{\beta^{2}} r\right) \mathfrak{J}_{n}\left(\frac{\omega}{\beta} r\right) y_{n, j}^{(1)}(\xi) \\
= & \left(\frac{n+1}{2 n+1} \mathfrak{J}_{n-1}\left(\frac{\omega}{\beta} r\right)-\frac{n}{2 n+1} \mathfrak{J}_{n+1}\left(\frac{\omega}{\beta} r\right)\right) \sqrt{n(n+1)} y_{n, j}^{(2)}(\xi) \\
& +\frac{\beta}{\omega} \cdot \frac{n(n+1)}{r} \mathfrak{J}_{n}\left(\frac{\omega}{\beta} r\right) y_{n, j}^{(1)}(\xi) ; \quad n \in \mathbb{N}, j \in\{1, \ldots, 2 n+1\} ;
\end{aligned}
$$

$\omega \in \mathbb{R} \backslash\{0\}, r \in] 0, \sigma], \xi \in \Omega$.

The introduction of certain boundary conditions on the stress yields so-called frequency equations. The attention is restricted here for physical reasons to the case $\omega>$ 0 . Those equations only allow discrete frequencies $\left\{{ }_{k} \omega_{n}^{\mathrm{T}}\right\}_{k \in \mathbb{N}_{0}, n \in \mathbb{N}}$ and $\left\{{ }_{k} \omega_{n}^{\mathrm{S}}\right\}_{k, n \in \mathbb{N}_{0}}$ for the toroidal and the spheroidal oscillations, respectively, where $k=0$ represents the fundamental mode and $k \geq 1$ stands for the $k$-th overtone. In seismology $k$ is called radial mode number and $n$ is called order of the oscillation or colatitudinal mode number. For further details on the eigenfrequencies and the derivation of the frequency equations we refer to [6]. As a consequence of those results we can use the set

$$
\left\{\mathfrak{l}_{n, j}\left({ }_{k} \omega_{n}^{\mathrm{S}}, \cdot\right)\right\}_{k, n \in \mathbb{N}_{0}} \cup\left\{\mathfrak{m}_{n, j}\left({ }_{k} \omega_{n}^{\mathrm{T}}, \cdot\right), \mathfrak{n}_{n, j}\left({ }_{k} \omega_{n}^{\mathrm{S}}, \cdot\right)\right\}_{k \in \mathbb{N}_{0}, n \in \mathbb{N}}
$$

as basis system for the eigenoscillations of the Earth.

We will now prove inequalities for the $\mathrm{l}^{2}\left(\overline{S_{\text {int }}}\right)-$ norms of the fundamental solutions for $\omega>0$. 
Lemma 4.7. The fundamental solutions satisfy the inequalities

$$
\begin{aligned}
&\left\|\mathfrak{l}_{n, j}(\omega, \cdot)\right\|_{1^{2}\left(\overline{S_{\mathrm{int}}}\right)}^{2} \leq \frac{\pi \alpha}{8 \omega}\left(\frac{1}{2 n+1}+4 \sigma+2 \sigma^{2}\right)+\frac{\pi \alpha^{3}}{2 \omega^{3}} \cdot \frac{n(n+1)}{2 n+1}, \\
&\left\|\mathfrak{m}_{n, j}(\omega, \cdot)\right\|_{1^{2}\left(\overline{S_{\mathrm{int}}}\right)}^{2} \leq \frac{\pi \beta \sigma^{2}}{8 \omega}, \\
&\left\|\mathfrak{n}_{n, j}(\omega, \cdot)\right\|_{1^{2}\left(\overline{S_{\mathrm{int}}}\right) \leq} \leq \frac{\omega^{2} \sigma^{5}}{5 \beta^{2}}+\frac{\pi}{48}\left(24 \sigma+51 \sigma^{2}+44 \sigma^{3}+18 \sigma^{4}\right) \frac{\omega}{\beta} \\
&+\frac{\pi}{48}\left(114 \sigma+81 \sigma^{2}+44 \sigma^{3}\right)+\frac{\pi}{16}\left(38 \sigma+17 \sigma^{2}\right) \frac{\beta}{\omega} \\
&+\frac{49 \pi \beta}{32 \omega(2 n+1)}+\frac{\pi \beta^{2}}{8 \omega^{2}}\left(\frac{\omega}{\beta}+4\right) \frac{n(n+1)}{2 n+1} \\
&+n(n+1) \frac{\beta^{2}}{\omega^{2}}\left(\sigma(1+\pi)+\frac{\pi \omega}{4 \beta}\left(2 \sigma+\sigma^{2}\right)\right),
\end{aligned}
$$

$\omega \in \mathbb{R}^{+}, n \in \mathbb{N}, j \in\{1, \ldots, 2 n+1\}$, where $n \geq 2$ in case of $\mathfrak{n}_{n, j}$.

Proof. In literature (see e.g. [30], p. 406) one finds the inequalities

$$
\left|J_{\nu}(x)\right| \leq 1, \quad\left|J_{\nu+1}(x)\right| \leq \frac{1}{\sqrt{2}}
$$

for $\nu>0$ and $x \in \mathbb{R}^{+}$. Consequently, the $\mathrm{l}^{2}\left(\overline{S_{\text {int }}}\right)$-norm of the toroidal basis functions allows the following estimate

$$
\begin{aligned}
\left\|\mathfrak{m}_{n, j}(\omega, \cdot)\right\|_{1^{2}\left(\overline{S_{\mathrm{int}}}\right)}^{2} & =\int_{0}^{\sigma} r^{2}\left(\mathfrak{J}_{n}\left(\frac{\omega}{\beta} r\right)\right)^{2} d r \int_{\Omega}\left(y_{n, j}^{(3)}(\xi)\right) \cdot\left(y_{n, j}^{(3)}(\xi)\right) d \omega(\xi) \\
& =\frac{\pi \beta}{2 \omega} \int_{0}^{\sigma} r\left(J_{n+1 / 2}\left(\frac{\omega}{\beta} r\right)\right)^{2} d r \leq \frac{\pi \beta \sigma^{2}}{8 \omega}
\end{aligned}
$$

for all $n \in \mathbb{N}$ and all $j \in\{1, \ldots, 2 n+1\}$.

According to [8], p. 12 the Bessel functions satisfy the differential relation

$$
J_{\nu-1}(x)-J_{\nu+1}(x)=2 J_{\nu}^{\prime}(x) ; \quad \nu, x \in \mathbb{R} ;
$$

such that we may replace the derivative in the representation of $\mathfrak{l}_{n, j}$ by

$\mathfrak{J}_{n}^{\prime}\left(\frac{\omega}{\alpha} r\right)=\left(\frac{\pi \alpha}{8 \omega}\right)^{1 / 2}\left(-r^{-3 / 2} J_{n+1 / 2}\left(\frac{\omega}{\alpha} r\right)+r^{-1 / 2}\left(J_{n-1 / 2}\left(\frac{\omega}{\alpha} r\right)-J_{n+3 / 2}\left(\frac{\omega}{\alpha} r\right)\right)\right)$

and obtain the identity

$\left\|\mathfrak{l}_{n, j}(\omega, \cdot)\right\|_{1^{2}\left(\overline{S_{\mathrm{int}}}\right)}^{2}=\int_{0}^{\sigma} r^{2}\left(\mathfrak{J}_{n}^{\prime}\left(\frac{\omega}{\alpha} r\right)\right)^{2}+\frac{\alpha^{2}}{\omega^{2}} n(n+1)\left(\mathfrak{J}_{n}\left(\frac{\omega}{\alpha} r\right)\right)^{2} d r$ 


$$
\begin{aligned}
= & \frac{\pi \alpha}{8 \omega} \int_{0}^{\sigma} r^{-1}\left(J_{n+1 / 2}\left(\frac{\omega}{\alpha} r\right)\right)^{2}-2 J_{n+1 / 2}\left(\frac{\omega}{\alpha} r\right)\left(J_{n-1 / 2}\left(\frac{\omega}{\alpha} r\right)-J_{n+3 / 2}\left(\frac{\omega}{\alpha} r\right)\right) \\
& +r\left(\left(J_{n-1 / 2}\left(\frac{\omega}{\alpha} r\right)\right)^{2}-2 J_{n-1 / 2}\left(\frac{\omega}{\alpha} r\right) J_{n+3 / 2}\left(\frac{\omega}{\alpha} r\right)+\left(J_{n+3 / 2}\left(\frac{\omega}{\alpha} r\right)\right)^{2}\right) d r \\
& +\frac{\pi \alpha^{3}}{2 \omega^{3}} n(n+1) \int_{0}^{\sigma} r^{-1}\left(J_{n+1 / 2}\left(\frac{\omega}{\alpha} r\right)\right)^{2} d r .
\end{aligned}
$$

A special case of the Weber-Schafheitlin integral is the identity (see e.g. [30], p. 405)

$$
\int_{0}^{\infty} t^{-1}\left(J_{\nu}(a t)\right)^{2} d t=\frac{1}{2 \nu} ; \quad a \in \mathbb{R}, \nu \in \mathbb{R}^{+}
$$

such that we find for $n \geq 1$ the inequality

$$
\left\|\mathfrak{l}_{n, j}(\omega, \cdot)\right\|_{1^{2}\left(\overline{\text { Snt }_{\text {int }}}\right)}^{2} \leq \frac{\pi \alpha}{8 \omega}\left(\frac{1}{2 n+1}+4 \sigma+2 \sigma^{2}\right)+\frac{\pi \alpha^{3}}{2 \omega^{3}} \cdot \frac{n(n+1)}{2 n+1} .
$$

The second derivative of the spherical Bessel function is given by

$$
\begin{aligned}
\frac{d^{2}}{d r^{2}} \mathfrak{J}_{n} & \left(\frac{\omega}{\beta} r\right) \\
= & \left(\frac{\pi \beta}{8 \omega}\right)^{1 / 2} \frac{\omega}{\beta}\left(\frac{3}{2} r^{-5 / 2} J_{n+1 / 2}\left(\frac{\omega}{\beta} r\right)\right. \\
& -\frac{1}{2} r^{-3 / 2}\left(\frac{\omega}{\beta}+1\right)\left(J_{n-1 / 2}\left(\frac{\omega}{\beta} r\right)-J_{n+3 / 2}\left(\frac{\omega}{\beta} r\right)\right) \\
& \left.+\frac{1}{2} r^{-1 / 2} \frac{\omega}{\beta}\left(J_{n-3 / 2}\left(\frac{\omega}{\beta} r\right)-2 J_{n+1 / 2}\left(\frac{\omega}{\beta} r\right)+J_{n+5 / 2}\left(\frac{\omega}{\beta} r\right)\right)\right) .
\end{aligned}
$$

Using this identity we are now able to calculate an upper bound for the $1^{2}\left(\overline{S_{\text {int }}}\right)-$ norm of $\mathfrak{n}_{n, j}$.

$$
\begin{aligned}
\frac{\omega^{2}}{\beta^{2}}\left\|\mathfrak{n}_{n, j}(\omega, \cdot)\right\|_{1^{2}}^{2}\left(\overline{S_{\mathrm{int}}}\right) \\
=\int_{0}^{\sigma} r^{4}\left(\frac{d^{2}}{d r^{2}} \mathfrak{J}_{n}\left(\frac{\omega}{\beta} r\right)\right)^{2}+(4+n(n+1)) r^{2}\left(\frac{d}{d r} \mathfrak{J}_{n}\left(\frac{\omega}{\beta} r\right)\right)^{2} d r \\
\quad+\int_{0}^{\sigma}\left(\frac{\omega^{4} r^{4}}{\beta^{4}}+n(n+1)\right)\left(\mathfrak{J}_{n}\left(\frac{\omega}{\beta} r\right)\right)^{2} d r \\
\quad+\int_{0}^{\sigma} 4 r^{3}\left(\frac{d^{2}}{d r^{2}} \mathfrak{J}_{n}\left(\frac{\omega}{\beta} r\right)\right)\left(\frac{d}{d r} \mathfrak{J}_{n}\left(\frac{\omega}{\beta} r\right)\right)+2 r^{4} \frac{\omega^{2}}{\beta^{2}} \mathfrak{J}_{n}\left(\frac{\omega}{\beta} r\right) \frac{d^{2}}{d r^{2}} \mathfrak{J}_{n}\left(\frac{\omega}{\beta} r\right) d r \\
\quad+\int_{0}^{\sigma}\left(4 \frac{\omega^{2} r^{3}}{\beta^{2}}+2 n(n+1) r\right) \mathfrak{J}_{n}\left(\frac{\omega}{\beta} r\right) \frac{d}{d r} \mathfrak{J}_{n}\left(\frac{\omega}{\beta} r\right) d r
\end{aligned}
$$




$$
\begin{aligned}
& \leq \int_{0}^{\sigma} \frac{\pi \omega}{8 \beta}\left(\frac{9}{4} r^{-1}\left(J_{n+1 / 2}\left(\frac{\omega}{\beta} r\right)\right)^{2}+3\left(\frac{\omega}{\beta}+1\right)\right. \\
& \left.+r\left(\frac{\omega}{\beta}+1\right)^{2}+4 \frac{\omega^{2}}{\beta^{2}} r^{3}+3 \frac{\omega}{\beta} r+2 \frac{\omega}{\beta}\left(\frac{\omega}{\beta}+1\right) r^{2}\right) d r \\
& +(4+n(n+1)) \frac{\pi \omega}{8 \beta}\left(\frac{1}{2 n+1}+4 \sigma+2 \sigma^{2}\right)+\frac{\omega^{4} \sigma^{5}}{5 \beta^{4}}+n(n+1) \sigma \\
& +\frac{\pi \omega}{2 \beta} \int_{0}^{\sigma} \frac{3}{2} r^{-1}\left(J_{n+1 / 2}\left(\frac{\omega}{\beta} r\right)\right)^{2}+3+\frac{\omega}{\beta}+1+2\left(\frac{\omega}{\beta}+1\right) r+2 \frac{\omega}{\beta} r+4 \frac{\omega}{\beta} r^{2} d r \\
& +\frac{\pi \beta}{2 \omega} \frac{\omega^{3}}{\beta^{3}} \int_{0}^{\sigma} \frac{3}{2} r+\left(\frac{\omega}{\beta}+1\right) r^{2}+2 \frac{\omega}{\beta} r^{3} d r+4 \frac{\omega^{3}}{\beta^{3}} \frac{\pi \beta}{4 \omega} \int_{0}^{\sigma} r+2 r^{2} d r \\
& +2 n(n+1) \frac{\pi \beta}{4 \omega} \frac{\omega}{\beta}\left(\int_{0}^{\sigma} r^{-1}\left(J_{n+1 / 2}\left(\frac{\omega}{\beta} r\right)\right)^{2} d r+2 \sigma\right) \\
& \leq \frac{9 \pi \omega}{32 \beta(2 n+1)}+\frac{\pi \omega}{8 \beta}\left(3\left(\frac{\omega}{\beta}+1\right) \sigma+\frac{\sigma^{2}}{2}\left(\frac{\omega}{\beta}+1\right)^{2}\right. \\
& \left.+\sigma^{4} \frac{\omega^{2}}{\beta^{2}}+\frac{3 \sigma^{2}}{2} \frac{\omega}{\beta}+\frac{2 \sigma^{3}}{3} \frac{\omega}{\beta}\left(\frac{\omega}{\beta}+1\right)\right) \\
& +(4+n(n+1)) \frac{\pi \omega}{8 \beta}\left(\frac{1}{2 n+1}+4 \sigma+2 \sigma^{2}\right)+\frac{\omega^{4} \sigma^{5}}{5 \beta^{4}}+n(n+1) \sigma \\
& +\frac{3 \omega \pi}{4 \beta(2 n+1)}+\frac{\pi \omega^{2}}{2 \beta^{2}}\left(\left(4+\frac{\omega}{\beta}\right) \sigma+\left(1+2 \frac{\omega}{\beta}\right) \sigma^{2}+\frac{4}{3} \frac{\omega}{\beta} \sigma^{3}\right) \\
& +\frac{\pi \omega^{2}}{2 \beta^{2}}\left(\frac{3}{4} \sigma^{2}+\left(\frac{\omega}{\beta}+1\right) \frac{\sigma^{3}}{3}+\frac{\omega}{\beta} \frac{\sigma^{4}}{2}\right)+\pi \frac{\omega^{2}}{\beta^{2}}\left(\frac{\sigma^{2}}{2}+\frac{2}{3} \sigma^{3}\right) \\
& +\frac{\pi n(n+1)}{2(2 n+1)}+n(n+1) \pi \sigma \\
& =\frac{\omega^{4} \sigma^{5}}{5 \beta^{4}}+\frac{\pi}{48}\left(24 \sigma+51 \sigma^{2}+44 \sigma^{3}+18 \sigma^{4}\right) \frac{\omega^{3}}{\beta^{3}}+\frac{\pi}{48}\left(114 \sigma+81 \sigma^{2}+44 \sigma^{3}\right) \frac{\omega^{2}}{\beta^{2}} \\
& +\frac{\pi}{16}\left(38 \sigma+17 \sigma^{2}\right) \frac{\omega}{\beta}+\frac{49 \pi \omega}{32 \beta(2 n+1)} \\
& +\frac{\pi}{8}\left(\frac{\omega}{\beta}+4\right) \frac{n(n+1)}{2 n+1}+n(n+1)\left(\sigma(1+\pi)+\frac{\pi \omega}{4 \beta}\left(2 \sigma+\sigma^{2}\right)\right) \text {. }
\end{aligned}
$$

The most important consequence of this lemma is the behaviour

$\left\|\mathfrak{l}_{n, j}(\omega, \cdot)\right\|_{1^{2}\left(\overline{S_{\mathrm{int}}}\right)}=O\left(n^{1 / 2}\right), \quad\left\|\mathfrak{m}_{n, j}(\omega, \cdot)\right\|_{1^{2}\left(\overline{S_{\mathrm{int}}}\right)}=O(1), \quad\left\|\mathfrak{l}_{n, j}(\omega, \cdot)\right\|_{1^{2}\left(\overline{S_{\mathrm{int}}}\right)}=O(n)$ as $n \rightarrow \infty$. 
Since the fundamental system consists of solutions for the Fourier transformed CauchyNavier equation we will now calculate the inverse Fourier transforms of those functions.

Theorem 4.8. The inverse Fourier transforms of the Hansen basis vectors are given by

$$
\begin{aligned}
& \mathcal{F}^{-1}\left(\mathfrak{l}_{n, j}(\cdot, r \xi)\right)(t) \\
&=\left(-\frac{1}{2}\left(\frac{\alpha}{r}\right)^{2}(-i)^{n+1} t P_{n}\left(-\frac{\alpha}{r} t\right) y_{n, j}^{(1)}(\xi)\right. \\
&\left.\quad+(-1)^{n} \frac{\alpha}{2 r} i^{n-1} P_{n}\left(-\frac{\alpha}{r} t\right) \sqrt{n(n+1)} y_{n, j}^{(2)}(\xi)\right) \chi_{]-\frac{r}{\alpha}, \frac{r}{\alpha}[}(t), \quad|t| \neq \frac{r}{\alpha}, \\
& \mathcal{F}^{-1}\left(\mathfrak{m}_{n, j}(\cdot, r \xi)\right)(t)=\frac{\beta}{2 r}(-i)^{n} P_{n}\left(-\frac{\beta}{r} t\right) \chi_{]-\frac{r}{\beta}, \frac{r}{\beta}[}(t) y_{n, j}^{(3)}(\xi), \quad|t| \neq \frac{r}{\beta}, \\
& \mathcal{F}^{-1}\left(\mathfrak{n}_{n, j}(\cdot, r \xi)\right)(t) \\
&=\quad\left(\frac{(-i)^{n-1} \beta}{(2 n+1) 2 r}\left((n+1) P_{n-1}\left(-\frac{\beta}{r} t\right)+n P_{n+1}\left(-\frac{\beta}{r} t\right)\right) \sqrt{n(n+1)} y_{n, j}^{(2)}(\xi)\right. \\
&\left.\quad+\frac{1}{2}(-1)^{n} n(n+1) \frac{\beta}{r} i^{n-1} P_{n}\left(-\frac{\beta}{r} t\right) y_{n, j}^{(1)}(\xi)\right) \chi_{]-\frac{r}{\beta}, \frac{r}{\beta}[}(t), \quad|t| \neq \frac{r}{\beta},
\end{aligned}
$$

where $P_{n}$ is the Legendre polynomial of degree $n$ and $\chi_{D}: \mathbb{R} \rightarrow \mathbb{R}$ is the characteristic function, defined by $\chi_{D}(t):=1$ if $t \in D$ and $\chi_{D}(t):=0$ if $t \notin D$. The identities are valid for $j \in\{1, \ldots, 2 n+1\}$, where $n \in \mathbb{N}_{0}$ in case of $\mathfrak{l}_{n, j}$, and $n \in \mathbb{N}$ in case of $\mathfrak{m}_{n, j}$ and $\mathfrak{n}_{n, j}$.

Proof. The inverse Fourier transforms of the spherical Bessel functions are calculated by

$$
\begin{aligned}
\mathcal{F}^{-1}\left(\mathfrak{J}_{n}\left(\frac{\dot{\gamma}}{\gamma}\right)\right)(t) & =\left(\frac{\pi \gamma}{2 r}\right)^{1 / 2} \frac{1}{2 \pi} \int_{-\infty}^{+\infty} \omega^{-1 / 2} J_{n+1 / 2}\left(\frac{\omega}{\gamma} r\right) e^{-i \omega t} d \omega \\
& =\left(\frac{\pi \gamma}{2 r}\right)^{1 / 2}\left(\frac{\gamma}{r}\right)^{1 / 2} \frac{1}{2 \pi} \int_{-\infty}^{+\infty} \tilde{\omega}^{-1 / 2} J_{n+1 / 2}(\tilde{\omega}) e^{i \tilde{\omega}(-\gamma t / r)} d \tilde{\omega}
\end{aligned}
$$

In [25], p. 206 we find the value of this integral and get

$$
\mathcal{F}^{-1}\left(\mathfrak{J}_{n}\left(\frac{\cdot}{\gamma} r\right)\right)(t)=\frac{\gamma}{2 r}(-i)^{n} P_{n}\left(-\frac{\gamma}{r} t\right) \chi_{]-\frac{r}{\gamma}, \frac{r}{\gamma}[}(t), \quad|t| \neq \frac{r}{\gamma} .
$$

Moreover, the inverse Fourier transforms of $\mathfrak{J}_{n}^{\prime}(\omega r / \alpha)$ and $(1 / \omega) \mathfrak{J}_{n}(\omega r / \gamma)$ have to be determined. For the first function we use the fact that (14) and $\left|J_{n+1 / 2}(-x)\right|=$ $\left|J_{n+1 / 2}(x)\right|$ imply $\lim _{\omega \rightarrow \pm \infty} \mathfrak{J}_{n}(\omega r / \alpha)=0$. With elementary calculations we obtain

$$
\mathcal{F}^{-1}\left(\mathfrak{J}_{n}^{\prime}\left(\frac{\dot{ }}{\alpha} r\right)\right)(t)=\frac{\alpha}{r} i t \mathcal{F}^{-1}\left(\mathfrak{J}_{n}\left(\frac{\dot{ }}{\alpha} r\right)\right)(t)
$$




$$
\begin{aligned}
& =-\frac{1}{2}\left(\frac{\alpha}{r}\right)^{2}(-i)^{n+1} t P_{n}\left(-\frac{\alpha}{r} t\right) \chi_{-\frac{r}{\alpha}, \frac{r}{\alpha}[}(t), \quad|t| \neq \frac{r}{\alpha}, \\
\mathcal{F}^{-1}\left(\frac{1}{\cdot} \mathfrak{J}_{n}\left(\frac{\dot{-}}{\gamma}\right)\right)(t) & =\left(\frac{\pi \gamma}{2 r}\right)^{1 / 2} \frac{1}{2 \pi} \int_{-\infty}^{+\infty} \omega^{-3 / 2} J_{n+1 / 2}\left(\frac{\omega}{\gamma} r\right) e^{-i \omega t} d \omega \\
& =\left(\frac{\pi \gamma}{2 r}\right)^{1 / 2} \frac{1}{2 \pi}\left(\frac{\gamma}{r}\right)^{-1 / 2} \int_{-\infty}^{+\infty} \tilde{\omega}^{-3 / 2} J_{n+1 / 2}(\tilde{\omega}) e^{i \tilde{\omega}(-\gamma t / r)} d \tilde{\omega}
\end{aligned}
$$

where the last integral can also be found in [25], p. 206. We get for $n \geq 1$

$$
\begin{aligned}
\mathcal{F}^{-1} & \left(\frac{1}{\cdot} \mathfrak{J}_{n}\left(\frac{\dot{\gamma}}{\gamma} r\right)\right)(t) \\
& =\left(\frac{\pi}{2}\right)^{1 / 2} \frac{1}{2 \pi}(-i)^{n-1} \frac{(n-1) !}{\Gamma(n+1)}(2 \pi)^{1 / 2} 2^{-1}\left(1-\left(\frac{\gamma}{r} t\right)^{2}\right) P_{n-1}^{(1,1)}\left(-\frac{\gamma}{r} t\right) \chi_{-\frac{r}{\gamma}, \frac{r}{\gamma}}[(t) \\
& =\frac{(-i)^{n-1}}{4 n}\left(1-\left(\frac{\gamma}{r} t\right)^{2}\right) P_{n-1}^{(1,1)}\left(-\frac{\gamma}{r} t\right) \chi_{-\frac{r}{\gamma}, \frac{r}{\gamma}}(t), \quad|t| \neq \frac{r}{\gamma},
\end{aligned}
$$

where $P_{n}^{(\nu, \mu)}$ is a Jacobi polynomial, given by $P_{n}^{(\nu, \mu)}(x)=(-1)^{n} 2^{-n}(n !)^{-1}(1-x)^{-\alpha}(1+x)^{-\beta} \frac{d^{n}}{d x^{n}}\left((1-x)^{\alpha+n}(1+x)^{\beta+n}\right), \quad|x|<1$,

such that

$$
\begin{aligned}
\mathcal{F}^{-1}\left(\frac{1}{\cdot} \mathfrak{J}_{n}\left(\frac{\dot{\gamma}}{\gamma}\right)\right)(t) & =\left.i^{n-1} 2^{-n-1}(n !)^{-1}\left(\frac{d^{n}}{d x^{n}}\left(\left(1-x^{2}\right)^{n}\right)\right)\right|_{x=-\gamma t / r} \chi_{-\frac{r}{\gamma}, \frac{r}{\gamma}}[(t) \\
& =(-1)^{n} i^{n-1} \frac{1}{2} P_{n}\left(-\frac{\gamma}{r} t\right) \chi_{-\frac{r}{\gamma}, \frac{r}{\gamma}}[t), \quad|t| \neq \frac{r}{\gamma} .
\end{aligned}
$$

To simplify notations we will write $\hat{\mathfrak{l}}_{n, j}(t, r \xi):=\mathcal{F}^{-1}\left(\mathfrak{l}_{n, j}(\cdot, r \xi)\right)(t)$ and so on. The obtained functions are elements of $\mathrm{L}^{2}\left(\mathbb{R} \times \overline{S_{\text {int }}}, \mathbb{C}^{3}\right)=: 1^{2}\left(\mathbb{R} \times \overline{S_{\text {int }}}\right)$. Note that $\mathfrak{l}_{n, j}$, $\mathfrak{m}_{n, j}$, and $\mathfrak{n}_{n, j}$ may be regarded as tempered distributions, such that $\hat{\mathfrak{l}}_{n, j}, \hat{\mathfrak{m}}_{n, j}$, and $\hat{\mathfrak{n}}_{n, j}$ represent (weak) solutions of the wave equations

$$
\Delta_{x} u-\frac{1}{\gamma^{2}} \frac{\partial^{2}}{\partial t^{2}} u=0, \quad \gamma \in\{\alpha, \beta\}
$$

(see e.g. [29]). A well-known property (see e.g. [10]) is the $\mathrm{L}^{2}([-1,1])$-orthogonality of the Legendre polynomials with $\left\|P_{n}\right\|_{\mathrm{L}^{2}([-1,1])}^{2}=2 /(2 n+1)$. This allows us to further investigate the $\mathrm{l}^{2}\left(\mathbb{R} \times \overline{S_{\mathrm{int}}}\right)$-norm of the fundamental system (in the time-space representation). 
Theorem 4.9. The $\mathrm{l}^{2}\left(\mathbb{R} \times \overline{S_{\mathrm{int}}}\right)$-norms of the inverse Fourier transforms of the fundamental solutions have the following properties:

$$
\begin{aligned}
\left\|\hat{\mathfrak{l}}_{n, j}\right\|_{1^{2}\left(\mathbb{R} \times \overline{S_{\mathrm{int}}}\right)}^{2}= & \frac{\alpha \sigma^{2}}{4}\left(\left(\frac{n+1}{2 n+1}\right)^{2} \frac{1}{2 n+3}+\left(\frac{n}{2 n+1}\right)^{2} \frac{1}{2 n-1}+\frac{n(n+1)}{2 n+1}\right) ; \\
& n \in \mathbb{N}_{0}, j \in\{1, \ldots, 2 n+1\} ; \\
\left\|\hat{\mathfrak{m}}_{n, j}\right\|_{1^{2}\left(\mathbb{R} \times \overline{S_{\mathrm{int}}}\right)}^{2 n} & \frac{\beta \sigma^{2}}{4(2 n+1)} ; \quad n \in \mathbb{N}, j \in\{1, \ldots, 2 n+1\} ; \\
\left\|\hat{\mathfrak{n}}_{n, j}\right\|_{1^{2}\left(\mathbb{R} \times \overline{S_{\mathrm{int}}}\right)}^{2}= & \frac{\beta \sigma^{2}}{4} \cdot \frac{n(n+1)}{(2 n+1)^{2}}\left(\frac{(n+1)^{2}}{2 n-1}+\frac{n^{2}}{2 n+3}\right)+\frac{\beta}{4} \cdot \frac{n^{2}(n+1)^{2}}{2 n+1} \sigma^{2} ; \\
& n \in \mathbb{N}, j \in\{1, \ldots, 2 n+1\} .
\end{aligned}
$$

Proof. Note that the considered functions have, as functions of the time $t$, compact support. Moreover, a simple substitution allows us to use the properties of the Legendre polynomials on the interval $[-1,1]$. We obtain

$$
\begin{aligned}
\int_{\frac{S_{\mathrm{int}}}{} \int_{-\infty}^{+\infty}\left|\hat{\mathfrak{m}}_{n, j}(t, x)\right|^{2} d t d x} & =\frac{\beta^{2}}{4} \int_{0}^{\sigma} 1 \cdot \int_{-r / \beta}^{r / \beta}\left(P_{n}\left(-\frac{\beta}{r} t\right)\right)^{2} d t d r \\
& =\frac{\beta^{2}}{4} \int_{0}^{\sigma} \frac{r}{\beta} \int_{-1}^{1}\left(P_{n}(\tau)\right)^{2} d \tau d r=\frac{\beta \sigma^{2}}{4(2 n+1)}
\end{aligned}
$$

for the toroidal oscillations. Applying the well-known recurrence formula (see e.g. $[10])$

$$
(2 n+1) t P_{n}(t)=(n+1) P_{n+1}(t)+n P_{n-1}(t), \quad n \in \mathbb{N},
$$

we are able to calculate the integral

$$
\begin{aligned}
\int_{-r / \alpha}^{r / \alpha}\left(t P_{n}\left(-\frac{\alpha}{r} t\right)\right)^{2} d t & =\int_{-1}^{1}\left(-\frac{r}{\alpha} \tau P_{n}(\tau)\right)^{2} \frac{r}{\alpha} d \tau \\
& =\frac{r^{3}}{\alpha^{3}} \int_{-1}^{1}\left(\tau P_{n}(\tau)\right)^{2} d \tau \\
& =\frac{r^{3}}{\alpha^{3}} \int_{-1}^{1}\left(\frac{n+1}{2 n+1} P_{n+1}(\tau)+\frac{n}{2 n+1} P_{n-1}(\tau)\right)^{2} d \tau \\
& =\frac{r^{3}}{\alpha^{3}}\left(\left(\frac{n+1}{2 n+1}\right)^{2} \frac{2}{2 n+3}+\left(\frac{n}{2 n+1}\right)^{2} \frac{2}{2 n-1}\right)
\end{aligned}
$$

such that we find for the spheroidal oscillations

$$
\left\|\hat{\mathfrak{l}}_{n, j}\right\|_{1^{2}\left(\mathbb{R} \times \overline{S_{\mathrm{int}}}\right)}^{2}
$$




$$
\begin{aligned}
= & \frac{\alpha^{4}}{4} \int_{0}^{\sigma} r^{-2} \int_{-r / \alpha}^{r / \alpha}\left(t P_{n}\left(-\frac{\alpha}{r} t\right)\right)^{2} d t d r \\
& +\frac{\alpha^{2}}{4} \int_{0}^{\sigma} \int_{-r / \alpha}^{r / \alpha}\left(P_{n}\left(-\frac{\alpha}{r} t\right)\right)^{2} d t d r n(n+1) \\
= & \frac{\alpha}{4} \int_{0}^{\sigma} r d r\left(\left(\frac{n+1}{2 n+1}\right)^{2} \frac{2}{2 n+3}+\left(\frac{n}{2 n+1}\right)^{2} \frac{2}{2 n-1}\right) \\
= & \frac{\alpha \sigma^{2}}{4} \cdot\left(\frac{\alpha^{2}}{\alpha(2 n+1)} n(n+1)\right. \\
\left\|\hat{\mathfrak{n}}_{n, j}\right\|_{\mathbb{1}^{2}\left(\mathbb{R} \times \overline{S_{\mathrm{int}}}\right)}^{2}= & \frac{\beta^{2} n(n+1)}{4(2 n+1)^{2}} \int_{0}^{\sigma} \frac{1}{2 n} \int_{-1}^{1}(n+1)^{2}\left(P_{n-1}(\tau)\right)^{2}+n^{2}\left(P_{n+1}(\tau)\right)^{2} d \tau d r \\
& +\frac{\beta^{2}}{4} n^{2}(n+1)^{2} \int_{0}^{\sigma} \frac{r}{\beta} \int_{-1}^{1}\left(P_{n}(\tau)\right)^{2} d \tau d r \\
= & \frac{\beta \sigma^{2}}{4} \cdot \frac{n(n+1)}{(2 n+1)^{2}}\left(\frac{n(n+1)}{2 n+1}\right) \\
2 n+1 & \left.\frac{n^{2}}{2 n+3}\right)+\frac{\beta}{4} \cdot \frac{n^{2}(n+1)^{2}}{2 n+1} \sigma^{2}
\end{aligned}
$$

Thus, the fundamental solutions $\hat{\mathfrak{l}}_{n, j}, \hat{\mathfrak{m}}_{n, j}$, and $\hat{\mathfrak{n}}_{n, j}$ have the behaviour

$\left\|\hat{\mathfrak{l}}_{n, j}\right\|_{1^{2}\left(\mathbb{R} \times \overline{S_{\mathrm{int}}}\right)}=O\left(n^{1 / 2}\right), \quad\left\|\hat{\mathfrak{m}}_{n, j}\right\|_{1^{2}\left(\mathbb{R} \times \overline{S_{\mathrm{int}}}\right)}=O\left(1 / n^{1 / 2}\right), \quad\left\|\hat{\mathfrak{n}}_{n, j}\right\|_{1^{2}\left(\mathbb{R} \times \overline{S_{\mathrm{int}}}\right)}=O\left(n^{3 / 2}\right)$ as $n \rightarrow \infty$.

Theorem 4.10. The function system of Theorem 4.8 is orthogonal with respect to $1^{2}\left(\mathbb{R} \times \overline{S_{\text {int }}}\right)$.

Proof. The $\mathrm{l}^{2}(\Omega)$-orthogonality of the functions $y_{n, j}^{(i)}$ already allows us to show that most of the occurring terms vanish. It remains to discuss integrals of the following types

$$
\begin{gathered}
\int_{-r / \alpha}^{r / \alpha} t P_{n}\left(-\frac{\alpha}{r} t\right) P_{n}\left(-\frac{\beta}{r} t\right) d t \\
\int_{-r / \alpha}^{r / \alpha} P_{n}\left(-\frac{\alpha}{r} t\right) P_{n+k}\left(-\frac{\beta}{r} t\right) d t, \quad k \in\{-1,1\} .
\end{gathered}
$$

Clearly,

$$
\int_{-r / \alpha}^{r / \alpha} t P_{0}\left(-\frac{\alpha}{r} t\right) P_{0}\left(-\frac{\beta}{r} t\right) d t=\int_{-r / \alpha}^{r / \alpha} t d t=0 .
$$


With the recurrence formula (15) and the substitution $t \leftrightarrow-\frac{\alpha}{r} t$ we can reduce the discussion to the calculation of the integrals

$$
\int_{-1}^{1} P_{n}(t) P_{n+k}\left(\frac{\beta}{\alpha} t\right) d t, \quad k \in\{-1,1\} .
$$

According to e.g. [10] the Legendre polynomials satisfy the identities

$$
\begin{gathered}
\int_{-1}^{1} F(t) P_{n}(t) d t=\frac{1}{2^{n} n !} \int_{-1}^{1} F^{(n)}(t)\left(1-t^{2}\right)^{n} d t ; \quad F \in \mathrm{C}^{(n)}([-1,1]), n \in \mathbb{N}_{0} ; \\
P_{n}(t)=\sum_{s=0}^{[n / 2]}(-1)^{s} \frac{(2 n-2 s) !}{2^{n}(n-2 s) !(n-s) ! s !} t^{n-2 s}, \quad t \in[-1,1], n \in \mathbb{N}_{0} .
\end{gathered}
$$

Those relations allow us to calculate the integrals of interest.

$$
\int_{-1}^{1} P_{n}(t) P_{n+k}\left(\frac{\beta}{\alpha} t\right) d t=\frac{1}{2^{n} n !} \int_{-1}^{1}\left(1-t^{2}\right)^{n} P_{n+k}^{(n)}\left(\frac{\beta}{\alpha} t\right) d t .
$$

For $k=-1$ we immediately obtain

$$
\int_{-1}^{1} P_{n}(t) P_{n-1}\left(\frac{\beta}{\alpha} t\right) d t=0, \quad n \in \mathbb{N} .
$$

For $k=1$ we use

$$
P_{n+1}^{(n)}(t)=(-1)^{0} \frac{(2 n+2) !}{2^{n+1}((n+1) !)^{2}}(n+1) ! t^{1},
$$

such that we find

$$
\int_{-1}^{1} P_{n}(t) P_{n+1}\left(\frac{\beta}{\alpha} t\right) d t=\frac{(2 n+2) !}{2^{2 n+1}(n+1)(n !)^{2}} \int_{-1}^{1}\left(1-t^{2}\right)^{n} t d t=0,
$$

since the integrand is an odd function.

We will now normalize our basis.

Definition 4.11. We define the following functions in $\mathrm{l}^{2}\left(\mathbb{R} \times \overline{S_{\mathrm{int}}}\right)$ :

$$
\begin{aligned}
& u_{n, j}^{(1)}:=\left\|\hat{\mathfrak{l}}_{n, j}\right\|_{1^{2}\left(\mathbb{R} \times \overline{S_{\text {int }}}\right)}^{-1} \hat{\mathfrak{l}}_{n, j} \\
& u_{n, j}^{(2)}:=\left\|\hat{\mathfrak{n}}_{n, j}\right\|_{1^{2}\left(\mathbb{R} \times \overline{S_{\text {int }}}\right)}^{-\hat{\mathfrak{n}}_{n, j}} \\
& u_{n, j}^{(3)}:=\left\|\hat{\mathfrak{m}}_{n, j}\right\|_{1^{2}\left(\mathbb{R} \times \overline{S_{\text {int }}}\right)}^{-1} \hat{\mathfrak{m}}_{n, j} .
\end{aligned}
$$

Note that the upper indices (1) and (2) refer to spheroidal oscillations, whereas (3) corresponds to toroidal oscillations. 


\section{Multiresolution}

Regarding the eigenfrequencies ${ }_{k} \omega_{n}$ we can interpret the colatitudinal mode number $n$ as a parameter for the spatial resolution, since $n$ refers to the degree of the used spherical harmonics $Y_{n, j}$. On the other hand the radial mode number $k$ is the enumeration of the overtones with respect to a given order $n$ of the oscillation. The larger $k$ is the larger is the frequency ${ }_{k} \omega_{n}$. Therefore, we consider $k$ as a parameter for the temporal resolution.

An ordinary Fourier analysis would consist of a spectral decomposition of observed oscillations in accordance to the function basis (13). As a result we obtain the amount of the contribution of each discrete frequency on the whole Earth. However, we are not able to analyze local variations of the intensities of the oscillations, since the used basis functions have no space localization. Moreover, the calculation of a Fourier coefficient with respect to one frequency requires an integration over the whole time interval, such that temporal variations of the phenomemon are smoothed out by this method.

We will now introduce a multiscale approach for the analysis of the eigenoscillations of the Earth. The obtained function spaces allow space $(x)$ as well as 'momentum' $(n)$ localization and time $(t)$ as well as frequency $(k)$ localization. We follow a standard approach which has already been applied to certain geoscientific problems (see e.g. [16], [14], [10], [20], [21], [9], [22]), where certainly a series of adaptations to our problem have to be made.

Definition 5.1. A function $\varphi_{0}:[0, \infty[\rightarrow \mathbb{R}$ is called generator of a scaling function, if it is admissible, i.e.

$$
\sum_{n=0}^{\infty}\left(\varphi_{0}(n)\right)^{2} n<\infty,
$$

and satisfies the following conditions:

a) $\varphi_{0}(0)=1$.

b) $\varphi_{0}$ is monotonically decreasing.

c) $\varphi_{0}$ is continuous in 0 .

The dilation $\varphi_{J}:\left[0, \infty\left[\rightarrow \mathbb{R}, J \in \mathbb{N}\right.\right.$, of such a function $\varphi_{0}$ is defined by

$$
\varphi_{J}(x):=\varphi_{0}\left(2^{-J} x\right), \quad x \in \mathbb{R}_{0}^{+} .
$$

It is easy to verify that a dilated generator $\varphi_{J}$ also satisfies the properties of a generator of a scaling function. 
Definition 5.2. Let $\varphi_{0}:[0, \infty[\rightarrow \mathbb{R}$ be a generator of a scaling function. The corresponding scaling functions ${ }^{i} \Phi_{J}:\left(\mathbb{R} \times \overline{S_{\text {int }}}\right) \times\left(\mathbb{R} \times \overline{S_{\text {int }}}\right) \rightarrow \mathbb{C}^{3 \times 3}, J \in \mathbb{N}_{0}, i \in$ $\{1,2,3\}$, are defined by

$$
{ }^{i} \Phi_{J}:=\sum_{n=0_{i}}^{\infty} \sum_{j=1}^{2 n+1} \varphi_{J}(n) u_{n, j}^{(i)} \otimes \overline{u_{n, j}^{(i)}}
$$

in the sense of $\mathrm{L}^{2}\left(\left(\mathbb{R} \times \overline{S_{\text {int }}}\right) \times\left(\mathbb{R} \times \overline{S_{\text {int }}}\right), \mathbb{C}^{3 \times 3}\right)$, where $0_{1}:=0,0_{2}:=0_{3}:=1$.

The admissibility condition (16) guarantees the $\mathrm{L}^{2}\left(\left(\mathbb{R} \times \overline{S_{\text {int }}}\right) \times\left(\mathbb{R} \times \overline{S_{\text {int }}}\right), \mathbb{C}^{3 \times 3}\right)$ convergence of the sequences of functions

$$
\left\{\left((t, x),\left(t^{\prime}, x^{\prime}\right)\right) \mapsto \sum_{n=0_{i}}^{N} \sum_{j=1}^{2 n+1} \varphi_{J}(n) u_{n, j}^{(i)} \otimes \overline{u_{n, j}^{(i)}}\right\}_{N \in \mathbb{N}}
$$

whose limits are denoted by ${ }^{i} \Phi_{J}$.

Definition 5.3. Let $\varphi_{0}:[0, \infty[\rightarrow \mathbb{R}$ be a generator of a scaling function. Moreover, let $\psi_{0}:\left[0, \infty\left[\rightarrow \mathbb{R}, \tilde{\psi}_{0}:[0, \infty[\rightarrow \mathbb{R}\right.\right.$ be a pair of admissible functions that satisfy the refinement equation

$$
\psi_{0}(x) \tilde{\psi}_{0}(x)=\left(\varphi_{1}(x)\right)^{2}-\left(\varphi_{0}(x)\right)^{2}, \quad x \in \mathbb{R}_{0}^{+} .
$$

Then $\psi_{0}$ is called generator of the (to $\varphi_{0}$ corresponding) primal wavelets

$$
{ }^{i} \Psi_{J}:=\sum_{n=0_{i}}^{\infty} \sum_{j=1}^{2 n+1} \psi_{J}(n) u_{n, j}^{(i)} \otimes \overline{u_{n, j}^{(i)}}
$$

and $\tilde{\psi}_{0}$ is called generator of the (to $\varphi_{0}$ corresponding) dual wavelets

$$
{ }^{i} \tilde{\Psi}_{J}:=\sum_{n=0_{i}}^{\infty} \sum_{j=1}^{2 n+1} \tilde{\psi}_{J}(n) u_{n, j}^{(i)} \otimes \overline{u_{n, j}^{(i)}}
$$

(in the sense of $\left.\mathrm{L}^{2}\left(\left(\mathbb{R} \times \overline{S_{\text {int }}}\right) \times\left(\mathbb{R} \times \overline{S_{\text {int }}}\right), \mathbb{C}^{3 \times 3}\right)\right)$.

Definition 5.4. Let $\Theta, \Lambda \in \mathrm{L}^{2}\left(\left(\mathbb{R} \times \overline{S_{\text {int }}}\right) \times\left(\mathbb{R} \times \overline{S_{\text {int }}}\right), \mathbb{C}^{3 \times 3}\right)$ be arbitrary functions and $u \in 1^{2}\left(\mathbb{R} \times \overline{S_{\text {int }}}\right)$ be an arbitrary solution of (1). Then we define the convolutions

$$
\begin{aligned}
(\Theta * u)(t, x) & :=\int_{\mathbb{R}} \int_{\overline{S_{\mathrm{int}}}} \Theta((t, x),(s, y)) u(s, y) d y d s \\
(\Theta * \Lambda)\left((t, x),\left(t^{\prime}, x^{\prime}\right)\right) & :=\int_{\mathbb{R}} \int_{\overline{S_{\mathrm{int}}}}(\Theta((t, x),(s, y))){\overline{\Lambda\left(\left(t^{\prime}, x^{\prime}\right),(s, y)\right)}}^{\mathrm{T}} d y d s, \\
\Theta^{(k+1)} & :=\Theta^{(k)} * \Theta, k \in \mathbb{N}, \quad \Theta^{(1)}:=\Theta,
\end{aligned}
$$


$(t, x),\left(t^{\prime}, x^{\prime}\right) \in \mathbb{R} \times \overline{S_{\text {int }}}$.

Elements of $\mathrm{L}^{2}\left(\left(\mathbb{R} \times \overline{S_{\text {int }}}\right) \times\left(\mathbb{R} \times \overline{S_{\text {int }}}\right), \mathbb{C}^{3 \times 3}\right)$ are called kernels. Note that the convolution of a kernel and a vectorial function yields a vectorial function whereas the convolution of two kernels always yields a kernel.

Lemma 5.5. Let ${ }^{i} \Theta \in \mathrm{L}^{2}\left(\left(\mathbb{R} \times \overline{S_{\text {int }}}\right) \times\left(\mathbb{R} \times \overline{S_{\text {int }}}\right), \mathbb{C}^{3 \times 3}\right) ; i=1,2,3$; be kernels with the representation

$$
{ }^{i} \Theta=\sum_{n=0_{i}}^{\infty} \sum_{j=1}^{2 n+1}\left({ }^{i} \Theta\right)^{\wedge}(n) u_{n, j}^{(i)} \otimes \overline{u_{n, j}^{(i)}}
$$

where $\left\{\left({ }^{i} \Theta\right)^{\wedge}(n)\right\}_{n \geq 0} ; i=1,2,3$; are real sequences. Then the iterated kernel ${ }^{i} \Theta^{(2)}$ has the representation

$$
{ }^{i} \Theta^{(2)}=\sum_{n=0_{i}}^{\infty} \sum_{j=1}^{2 n+1}\left(\left({ }^{i} \Theta\right)^{\wedge}(n)\right)^{2} u_{n, j}^{(i)} \otimes \overline{u_{n, j}^{(i)}}
$$

in the sense of $\mathrm{L}^{2}\left(\left(\mathbb{R} \times \overline{S_{\text {int }}}\right) \times\left(\mathbb{R} \times \overline{S_{\text {int }}}\right), \mathbb{C}^{3 \times 3}\right)$.

Proof. Note that the convolutions can be interpreted as scalar products in $l^{2}\left(\mathbb{R} \times \overline{S_{\text {int }}}\right)$, such that we may apply Parseval's identity to obtain

$$
\begin{aligned}
& { }^{i} \Theta^{(2)}\left((t, x),\left(t^{\prime}, x^{\prime}\right)\right)=\int_{\mathbb{R} \times \overline{S_{\text {int }}}} \Theta((t, x),(s, y)){\overline{\Theta\left(\left(t^{\prime}, x^{\prime}\right),(s, y)\right)}}^{\mathrm{T}} d(s, y) \\
& =\int_{\mathbb{R} \times \overline{S_{\mathrm{int}}}} \Theta((t, x),(s, y)) \Theta\left((s, y),\left(t^{\prime}, x^{\prime}\right)\right) d(s, y) \\
& =\int_{\mathbb{R} \times \overline{S_{\mathrm{int}}}} \sum_{n, m=0_{i}}^{\infty} \sum_{j=1}^{2 n+1} \sum_{k=1}^{2 m+1}\left({ }^{i} \Theta\right)^{\wedge}(n)\left({ }^{i} \Theta\right)^{\wedge}(m) u_{n, j}^{(i)}(t, x){\overline{u_{n, j}^{(i)}(s, y)}}^{\mathrm{T}} \\
& \cdot u_{m, k}^{(i)}(s, y){\overline{u_{m, k}^{(i)}\left(t^{\prime}, x^{\prime}\right)}}^{\mathrm{T}} d(s, y) \\
& =\sum_{n=0_{i}}^{\infty} \sum_{j=1}^{2 n+1}\left(\left({ }^{i} \Theta\right)^{\wedge}(n)\right)^{2} u_{n, j}^{(i)}(t, x) \otimes \overline{u_{n, j}^{(i)}\left(t^{\prime}, x^{\prime}\right)}
\end{aligned}
$$

for almost every $\left((t, x),\left(t^{\prime}, x^{\prime}\right)\right) \in\left(\mathbb{R} \times \overline{S_{\text {int }}}\right)^{2}$. Since

$$
\sum_{n=0_{i}}^{\infty} \sum_{j=1}^{2 n+1}\left(\left({ }^{i} \Theta\right)^{\wedge}(n)\right)^{4} \leq \sum_{\substack{n=0_{i} \\\left({ }^{i} \Theta\right)^{\wedge}(n) \geq 1}}^{\infty}\left(\left({ }^{i} \Theta\right)^{\wedge}(n)\right)^{4}+\sum_{\substack{n=0_{i} \\\left({ }^{i} \Theta\right)^{\wedge}(n)<1}}^{\infty}\left(\left({ }^{i} \Theta\right)^{\wedge}(n)\right)^{2}
$$

and

$$
\sum_{n=0_{i}}^{\infty} \sum_{j=1}^{2 n+1}\left(\left({ }^{i} \Theta\right)^{\wedge}(n)\right)^{2}<+\infty
$$


${ }^{i} \Theta^{(2)}$ must be square-integrable.

Definition 5.6. The space $\mathrm{CN}$ is defined by

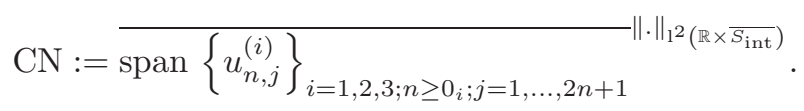

The subspaces of toroidal and spheroidal solutions are denoted by $\mathrm{CN}^{\mathrm{T}}$ and $\mathrm{CN}^{\mathrm{S}}$, respectively.

Note that the strong $l^{2}\left(\mathbb{R} \times \overline{S_{\text {int }}}\right)$-convergence of the series in $\mathrm{CN}$ implies that every element of $\mathrm{CN}$ consists of sums of weak solutions of the wave equations

$$
\Delta_{x} u-\frac{1}{\gamma^{2}} \frac{\partial^{2}}{\partial t^{2}} u=0, \quad \gamma \in\{\alpha, \beta\} .
$$

Since the wave equation is not elliptic we cannot conclude here that weak solutions are also strong, i.e. classical, solutions.

We will now show that the convolutions ${ }^{i} \Phi_{J}^{(2)} * u$ can be used for low-pass filtering of a function $u \in \mathrm{CN}$. Those convolutions

$$
{ }^{i} \Phi_{J}^{(2)} * u=\int_{\mathbb{R} \times \overline{S_{\mathrm{int}}}}{ }^{i} \Phi_{J}^{(2)}(\cdot,(t, x)) u(t, x) d(t, x)
$$

can be calculated numerically by an appropriate quadrature formula. If a bandlimited generator is chosen, i.e. a function $\varphi_{0}$ with compact support, then the kernel ${ }^{i} \Phi_{J}^{(2)}$ in (18) can be evaluated exactly. Note that a calculation of the tensors $u_{n, j}^{(i)} \otimes \overline{u_{n, j}^{(i)}}$ is not necessary since the corresponding expression in the integrand of (18) can be reduced to the multiplication of a vector with a euclidean scalar product:

$$
\left(u_{n, j}^{(i)} \otimes \overline{u_{n, j}^{(i)}}\right) u=u_{n, j}^{(i)}\left(u \cdot u_{n, j}^{(i)}\right) .
$$

Definition 5.7. Let $\varphi_{0}:[0, \infty[\rightarrow \mathbb{R}$ be a generator of a scaling function. Then we define the spheroidal and the toroidal scale spaces by

$$
\begin{aligned}
V_{J}^{\mathrm{S}} & :=\left\{\left(\left({ }^{1} \Phi_{J}\right)^{(2)}+\left({ }^{2} \Phi_{J}\right)^{(2)}\right) * u \mid u \in \mathrm{CN}\right\}, \\
V_{J}^{\mathrm{T}} & :=\left\{\left({ }^{3} \Phi_{J}\right)^{(2)} * u \mid u \in \mathrm{CN}\right\},
\end{aligned}
$$

respectively, and the spheroidal and the toroidal detail spaces by

$$
\begin{aligned}
W_{J}^{\mathrm{S}} & :=\left\{\left({ }^{1} \tilde{\Psi}_{J} *{ }^{1} \Psi_{J}+{ }^{2} \tilde{\Psi}_{J} *{ }^{2} \Psi_{J}\right) * u \mid u \in \mathrm{CN}\right\}, \\
W_{J}^{\mathrm{T}} & :=\left\{{ }^{3} \tilde{\Psi}_{J} *{ }^{3} \Psi_{J} * u \mid u \in \mathrm{CN}\right\} .
\end{aligned}
$$


The scale spaces form a multiresolution.

Theorem 5.8. For a given generator of a scaling function the corresponding scale spaces form a monotonically increasing sequence

$$
\begin{gathered}
V_{0}^{\mathrm{S}} \subset \cdots V_{J}^{\mathrm{S}} \subset V_{J+1}^{\mathrm{S}} \subset \cdots \subset \mathrm{CN}^{\mathrm{S}}, \\
V_{0}^{\mathrm{T}} \subset \cdots V_{J}^{\mathrm{T}} \subset V_{J+1}^{\mathrm{T}} \subset \cdots \subset \mathrm{CN}^{\mathrm{T}},
\end{gathered}
$$

and

$$
\bigcup_{J \in \mathbb{N}_{0}} V_{J}^{\mathrm{S}}\|\cdot\|_{1^{2}\left(\mathbb{R} \times \overline{\mathrm{S}_{\mathrm{int}}}\right)}=\mathrm{CN}^{\mathrm{S}}, \quad \bigcup_{J \in \mathbb{N}_{0}} V_{J}^{\mathrm{T}}\|\cdot\|_{1^{2}\left(\mathbb{R} \times \overline{\mathrm{S}_{\mathrm{int}}}\right)}=\mathrm{CN}^{\mathrm{T}},
$$

such that

$$
\overline{\bigcup_{J \in \mathbb{N}_{0}}\left(V_{J}^{\mathrm{S}} \oplus V_{J}^{\mathrm{T}}\right)} \|^{\|\|_{1^{2}}\left(\mathbb{R} \times \overline{\mathrm{S}_{\mathrm{int}}}\right)}=\mathrm{CN} .
$$

Moreover, an approximate identity is given:

$$
\lim _{J \rightarrow \infty}\left\|\sum_{i=1}^{3}{ }^{i} \Phi_{J}^{(2)} * u-u\right\|_{1^{2}\left(\mathbb{R} \times \overline{S_{\mathrm{int}}}\right)}=0
$$

for all $u \in \mathrm{CN}$.

Proof. For every $v \in \mathrm{CN}$ we find an orthogonal series

$$
v=\sum_{i=1}^{3} \sum_{n=0_{i}}^{\infty} \sum_{j=1}^{2 n+1} v^{\wedge}(i, n, j) u_{n, j}^{(i)}
$$

in the sense of $\mathrm{l}^{2}\left(\mathbb{R} \times \overline{S_{\text {int }}}\right)$. The convolution ${ }^{i} \Phi_{J}^{(2)} * v, J \in \mathbb{N}_{0}$ fixed, can, therefore, be expressed by the Parseval identity

$$
{ }^{i} \Phi_{J}^{(2)} * v=\sum_{n=0_{i}}^{\infty} \sum_{j=1}^{2 n+1}\left(\varphi_{J}(n)\right)^{2} v^{\wedge}(i, n, j) u_{n, j}^{(i)}, \quad i \in\{1,2,3\},
$$

with respect to $l^{2}\left(\mathbb{R} \times \overline{S_{\text {int }}}\right)$. Now let $w$ be given by the $l^{2}\left(\mathbb{R} \times \overline{S_{\text {int }}}\right)$-expansion

$$
w=\sum_{i=1}^{3} \sum_{n=0_{i}}^{\infty} \sum_{j=1}^{2 n+1} w^{\wedge}(i, n, j) u_{n, j}^{(i)},
$$

where

$$
w^{\wedge}(i, n, j):=\left\{\begin{array}{cl}
0 & \text { if } \varphi_{J+1}(n)=0 \\
\left(\frac{\varphi_{J}(n)}{\varphi_{J+1}(n)}\right)^{2} v^{\wedge}(i, n, j) & \text { if } \varphi_{J+1}(n) \neq 0
\end{array} .\right.
$$


Note that the monotonicity $\varphi_{J+1}(n) \geq \varphi_{J}(n)$ implies that $w \in \mathrm{CN}$ if $v \in \mathrm{CN}$. Clearly, ${ }^{i} \Phi_{J+1}^{(2)} * w$ is an element of the corresponding scale space of scale $J+1$. Moreover,

$$
\begin{aligned}
{ }^{i} \Phi_{J+1}^{(2)} * w & =\sum_{n=0_{i}}^{\infty} \sum_{j=1}^{2 n+1}\left(\varphi_{J+1}(n)\right)^{2} w^{\wedge}(i, n, j) u_{n, j}^{(i)} \\
& =\sum_{n=0_{i}}^{\infty} \sum_{j=1}^{2 n+1}\left(\varphi_{J}(n)\right)^{2} v^{\wedge}(i, n, j) u_{n, j}^{(i)} \\
& ={ }^{i} \Phi_{J}^{(2)} * v, \quad i \in\{1,2,3\},
\end{aligned}
$$

since $\varphi_{J+1}(n)=0$ implies that $\varphi_{J}(n)=0$. Thus, the monotonicity of the scale spaces has been proved.

For the approximate identity property we investigate the limit

$$
\lim _{J \rightarrow \infty}\left\|\sum_{i=1}^{3}{ }^{i} \Phi_{J}^{(2)} * v-v\right\|_{1^{2}\left(\mathbb{R} \times \overline{S_{\mathrm{int}}}\right)}^{2}=\sum_{i=1}^{3} \sum_{n=0_{i}}^{\infty} \sum_{j=1}^{2 n+1}\left(v^{\wedge}(i, n, j)\right)^{2}\left(\left(\varphi_{J}(n)\right)^{2}-1\right)^{2} .
$$

Since $v \in 1^{2}\left(\mathbb{R} \times \overline{S_{\text {int }}}\right)$ and $0 \leq 1-\left(\varphi_{J}(n)\right)^{2} \leq 1$, we may interchange the limit $J \rightarrow \infty$ with the series and get

$$
\lim _{J \rightarrow \infty}\left\|\sum_{i=1}^{3}{ }^{i} \Phi_{J}^{(2)} * v-v\right\|_{1^{2}\left(\mathbb{R} \times \overline{S_{\mathrm{int}}}\right)}^{2}=0 .
$$

An immediate consequence of the approximate identity property is that the unions of the scale spaces are closed in the corresponding spaces $\mathrm{CN}^{\mathrm{S}}, \mathrm{CN}^{\mathrm{T}}$, and $\mathrm{CN}$, respectively.

Moreover, the detail spaces represent the steps between consecutive scale spaces.

Theorem 5.9. For a given generator of a scaling function and given generators of the primal and the dual mother wavelets, respectively, the corresponding scale spaces and detail spaces satisfy

$$
V_{J+1}^{\mathrm{S}}=V_{J}^{\mathrm{S}}+W_{J}^{\mathrm{S}}, \quad V_{J+1}^{\mathrm{T}}=V_{J}^{\mathrm{T}}+W_{J}^{\mathrm{T}},
$$

where

$$
{ }^{i} \Phi_{J+1}^{(2)} * u={ }^{i} \Phi_{J}^{(2)} * u+{ }^{i} \tilde{\Psi}_{J} *{ }^{i} \Psi_{J} * u
$$

for all $J \in \mathbb{N}$ and all $u \in \mathrm{CN}$.

Proof. For $v \in \mathrm{CN}$ with

$$
v=\sum_{i=1}^{3} \sum_{n=0_{i}}^{\infty} \sum_{j=1}^{2 n+1} v^{\wedge}(i, n, j) u_{n, j}^{(i)}
$$


(in the sense of $\left.1^{2}\left(\mathbb{R} \times \overline{S_{\text {int }}}\right)\right)$ we get

$$
\begin{aligned}
{ }^{i} \Phi_{J}^{(2)} * v+{ }^{i} \tilde{\Psi}_{J} *{ }^{i} \Psi_{J} * v & =\sum_{n=0_{i}}^{\infty} \sum_{j=1}^{2 n+1}\left(\left(\varphi_{J}(n)\right)^{2}+\tilde{\psi}_{J}(n) \psi_{J}(n)\right) v^{\wedge}(i, n, j) u_{n, j}^{(i)} \\
& =\sum_{n=0_{i}}^{\infty} \sum_{j=1}^{2 n+1}\left(\varphi_{J+1}(n)\right)^{2} v^{\wedge}(i, n, j) u_{n, j}^{(i)} \\
& ={ }^{i} \Phi_{J+1}^{(2)} * v
\end{aligned}
$$

according to the refinement equation. This also implies that $V_{J+1}^{\mathrm{S}} \subset V_{J}^{\mathrm{S}}+W_{J}^{\mathrm{S}}$ and $V_{J+1}^{\mathrm{T}} \subset V_{J}^{\mathrm{T}}+W_{J}^{\mathrm{T}}$. Now let $u, v \in \mathrm{CN}$ be arbitrary. Moreover, let $w \in \mathrm{CN}$ be given by

$$
w^{\wedge}(i, n, j):=\left\{\begin{array}{cc}
\left(\left(\varphi_{J}(n)\right)^{2} u^{\wedge}(i, n, j)+\left(\left(\varphi_{J+1}(n)\right)^{2}-\left(\varphi_{J}(n)\right)^{2}\right) v^{\wedge}(i, n, j)\right)\left(\varphi_{J+1}(n)\right)^{-2}, \\
\text { if } \varphi_{J+1}(n) \neq 0 \\
0 & \text { if } \varphi_{J+1}(n)=0
\end{array}\right.
$$

It is easy to see that

$$
\begin{aligned}
{ }^{i} \Phi_{J+1}^{(2)} * w & =\sum_{n=0_{i}}^{\infty} \sum_{j=1}^{2 n+1}\left(\varphi_{J+1}(n)\right)^{2} w^{\wedge}(i, n, j) u_{n, j}^{(i)} \\
& =\sum_{n=0_{i}}^{\infty} \sum_{j=1}^{2 n+1}\left(\left(\varphi_{J}(n)\right)^{2} u^{\wedge}(i, n, j)+\psi_{J}(n) \tilde{\psi}_{J}(n) v^{\wedge}(i, n, j)\right) u_{n, j}^{(i)} \\
& ={ }^{i} \Phi_{J}^{(2)} * u+{ }^{i} \tilde{\Psi}_{J} *{ }^{i} \Psi_{J} * v .
\end{aligned}
$$

This implies $V_{J}^{\mathrm{S}}+W_{J}^{\mathrm{S}} \subset V_{J+1}^{\mathrm{S}}$ and $V_{J}^{\mathrm{T}}+W_{J}^{\mathrm{T}} \subset V_{J+1}^{\mathrm{T}}$.

Since the arguments of the scaling functions and the wavelets are eight-dimensional and the values are tensors, we need some simplifying assumptions for their visualization. First, we restrict our attention to a sphere of radius $R$ and use the addition theorem for vector spherical harmonics $([10])$, such that we find

$$
\begin{gathered}
{ }^{3} \Phi_{J}((t, R \xi),(\tau, R \eta)) \\
=\sum_{n=1}^{\infty} \sum_{j=1}^{2 n+1} \varphi_{J}(n) \frac{4(2 n+1)}{\beta \sigma^{2}} \frac{\beta^{2}}{4 R^{2}} P_{n}\left(-\frac{\beta}{R} t\right) P_{n}\left(-\frac{\beta}{R} \tau\right) y_{n, j}^{(3)}(\xi) \otimes y_{n, j}^{(3)}(\eta) \\
=\sum_{n=1}^{\infty} \varphi_{J}(n) \frac{(2 n+1)^{2} \beta}{4 \pi \sigma^{2} R^{2}} P_{n}\left(-\frac{\beta}{R} t\right) P_{n}\left(-\frac{\beta}{R} \tau\right) \frac{1}{n(n+1)} \cdot \\
\cdot\left(P_{n}^{\prime \prime}(\xi \cdot \eta)(\xi \wedge \eta) \otimes(\eta \wedge \xi)+P_{n}^{\prime}(\xi \cdot \eta)((\xi \cdot \eta) \mathrm{i}-\eta \otimes \xi)\right)
\end{gathered}
$$


for $t, \tau \in]-R / \beta, R / \beta[$ and $\xi, \eta \in \Omega$, where $\mathrm{i}$ is the identity tensor. The square of the euclidean norm of this tensor may be written as

$$
\begin{aligned}
\left({ }^{3} \Phi_{J}:{ }^{3} \Phi_{J}\right) & ((t, R \xi),(\tau, R \eta)) \\
=\sum_{n, m=1}^{\infty} & \varphi_{J}(n) \frac{(2 n+1)^{2} \beta}{4 \pi \sigma^{2} R^{2}} P_{n}\left(-\frac{\beta}{R} t\right) P_{n}\left(-\frac{\beta}{R} \tau\right) \frac{1}{n(n+1)} \varphi_{J}(m) \frac{(2 m+1)^{2} \beta}{4 \pi \sigma^{2} R^{2}} \\
& \cdot P_{m}\left(-\frac{\beta}{R} t\right) P_{m}\left(-\frac{\beta}{R} \tau\right) \frac{1}{m(m+1)} \cdot\left(P_{n}^{\prime \prime}(\xi \cdot \eta) P_{m}^{\prime \prime}(\xi \cdot \eta)|\xi \wedge \eta|^{4}\right. \\
& +P_{n}^{\prime}(\xi \cdot \eta) P_{m}^{\prime}(\xi \cdot \eta)\left(3(\xi \cdot \eta)^{2}+1-2(\xi \cdot \eta)^{2}\right) \\
& \left.+\left(P_{n}^{\prime \prime}(\xi \cdot \eta) P_{m}^{\prime}(\xi \cdot \eta)+P_{m}^{\prime \prime}(\xi \cdot \eta) P_{n}^{\prime}(\xi \cdot \eta)\right)\left(-(\xi \cdot \eta)|\xi \wedge \eta|^{2}-0\right)\right)
\end{aligned}
$$

Since $|\xi \wedge \eta|^{2}=1-(\xi \cdot \eta)^{2}$ for $\xi, \eta \in \Omega$, we may write this function in dependence of $t, \tau, R$, and the spherical distance $\xi \cdot \eta=: \delta$. An analogous result is obtained for the wavelets.

We will now choose $\tilde{\tau}:=\tau \beta / R$ fixed and plot the function

$$
\begin{aligned}
& F_{J}(\tilde{t}, \delta)=\sum_{n, m=1}^{2^{J}-1} \frac{(2 n+1)^{2}}{n(n+1)} \frac{(2 m+1)^{2}}{m(m+1)} P_{n}(-\tilde{t}) P_{n}(-\tilde{\tau}) P_{m}(-\tilde{t}) P_{m}(-\tilde{\tau}) \\
& \times\left(P_{n}^{\prime \prime}(\delta) P_{m}^{\prime \prime}(\delta)\left(1-\delta^{2}\right)^{2}+P_{n}^{\prime}(\delta) P_{m}^{\prime}(\delta)\left(1+\delta^{2}\right)+\left(P_{n}^{\prime \prime}(\delta) P_{m}^{\prime}(\delta)+P_{m}^{\prime \prime}(\delta) P_{n}^{\prime}(\delta)\right)\left(\delta^{3}-\delta\right)\right)
\end{aligned}
$$

$\tilde{t}=\frac{t \beta}{R}, \delta \in[-1,1]$; which refers to the Shannon scaling function given by

$$
\varphi_{0}(x):=\left\{\begin{array}{ll}
1, & \text { if } 0 \leq x<1 \\
0, & \text { if } x \geq 1
\end{array} .\right.
$$



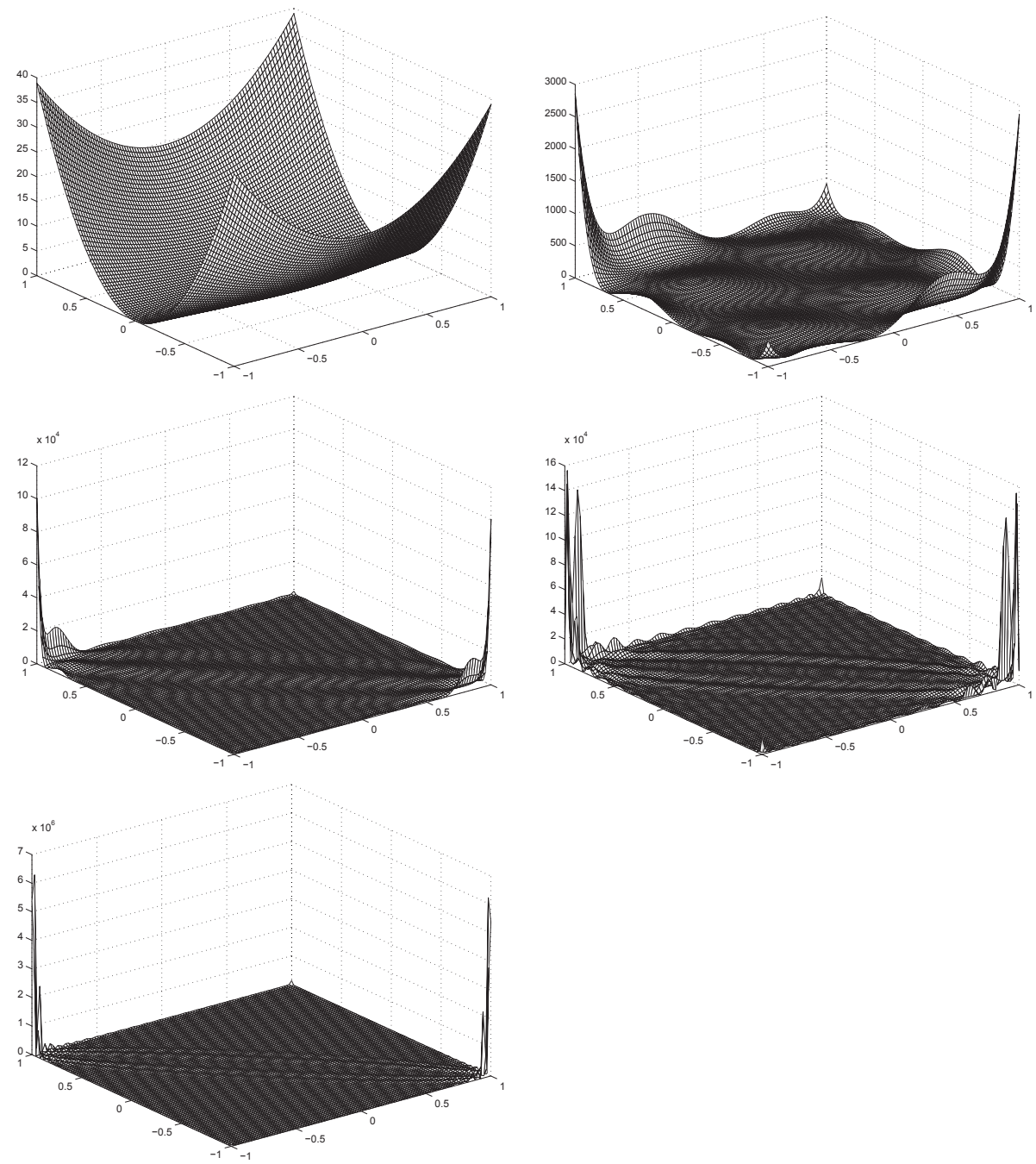

Figure 1: Visualization of toroidal Shannon scaling functions for $\tau=-0.98 R / \beta$ 

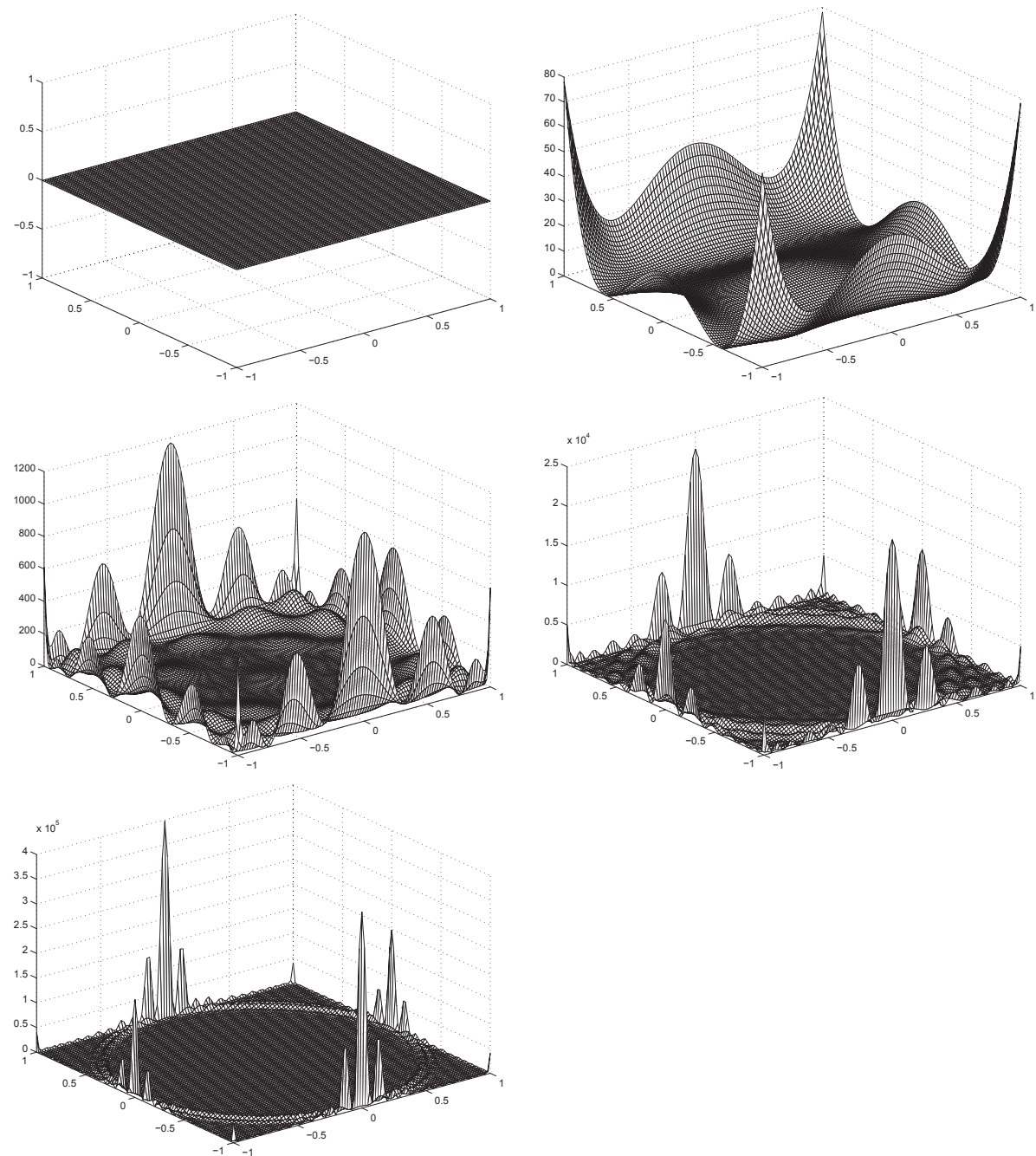

Figure 2: Visualization of toroidal Shannon scaling functions for $\tau=0$ 

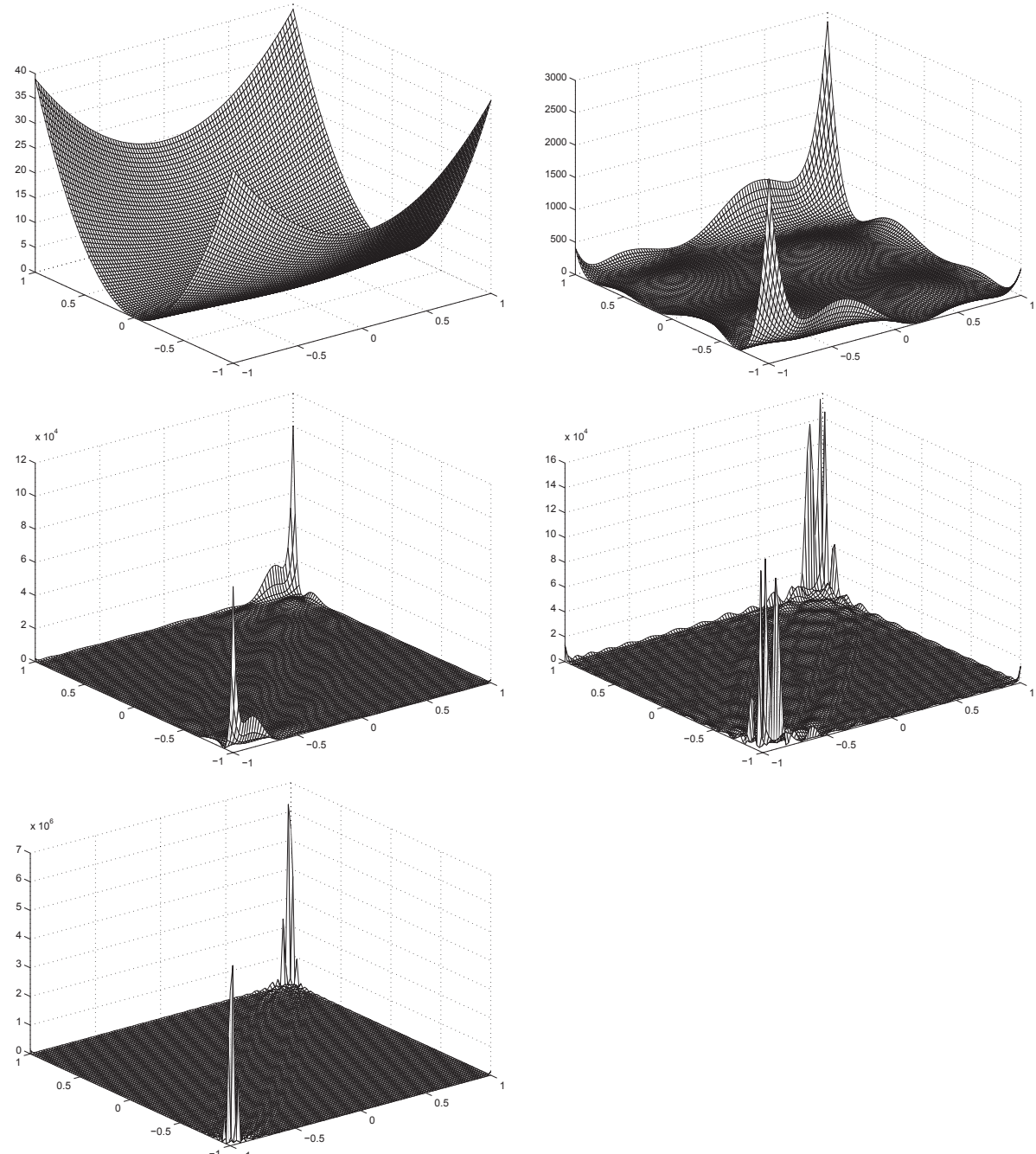

Figure 3: Visualization of toroidal Shannon scaling functions for $\tau=0.98 R / \beta$ 
Figure 1 shows $F_{J}$ for $J=1, \ldots, 5$ (in the order of reading) for $\tau=-0.98 R / \beta$. Finally, in Figures 2 and 3 the corresponding images for $\tau=0$ and $\tau=0.98 R / \beta$, respectively, are shown. Certainly, $F_{1}$ vanishes if $\tau=0$ is chosen. The right horizontal axis refers to the time $t$ and the left horizontal axis refers to the spherical distance $\delta$. Note the increasing space and time localizing character of the scaling functions as the scale increases. For $\tau=-0.98 R / \beta$ (Figure 1) we have a high peak at time $t=-1(R / \beta)$ and spherical distance $\delta=1$. Hence, the kernel concentrates on the effects near the observed position during a small time window. Since the plotted kernels are toroidal scaling functions we can think about a wave travelling along the sphere of radius $R$. The energy of this oscillation will be distributed in all directions over the surface. Finally, at a later point of time $(t \approx 1)$ the waves are focused at the antipode $(\delta=-1)$, see Figure 4 for an illustration. This is an explanation for the peak at the opposite end of the diagram.

If we choose $\tau=0$ (Figure 2) we find a concentration around this time. Moreover,

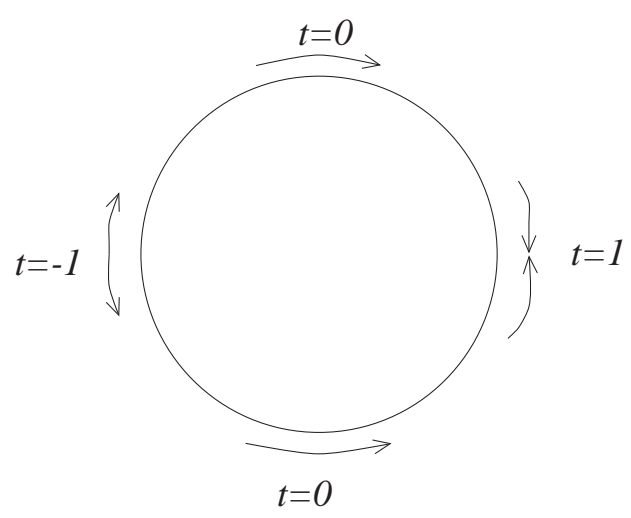

Figure 4: Exemplary path of a toroidal wave

note that our imagination of a travelling wave would include that this wave came from an orthogonal direction $(\delta=0)$, i.e. from the angle $-\pi / 2$, at time $t=-1(R / \beta)$ and will arrive on the other side, i.e. at angle $\pi / 2$ and the same spherical distance $\delta=0$, at time $t=1(R / \beta)$ or vice versa.

Finally at time $\tau=0.98 R / \beta$ (Figure 3 ) the concentration of the scaling function has moved near the time $t=1(R / \beta)$ for points in the neighbourhood $(\delta \approx 1)$, whereas a high peak is also found for the origin of the motion $(t=-1(R / \beta))$ at the antipode $(\delta \approx-1)$. 


\section{Conclusions}

A brief repetition of the mathematical derivation of the well-known Hansen vectors as fundamental solutions of the Cauchy-Navier equation describing the eigenoscillations of the Earth was given. Those well-known results were further analysed in this paper. A norm-estimate of the frequency dependent Hansen vectors was calculated. The inverse Fourier transform was applied to the fundamental system in the frequency space in order to obtain a time dependent system of functions. The derived function system has a compact domain with respect to the time, such that the oscillations in this model only have a finite (temporal) duration. Further properties of those functions were derived, namely their $\mathrm{l}^{2}$-orthogonality and a norm-estimate.

Based on the orthogonality of the derived functions in the space-time domain we constructed a multiresolution analysis for the toroidal and the spheroidal eigenoscillations. This allows a zooming into details of different (temporal and spatial) resolutions. An example of a scaling function was plotted. The graph demonstrates that the multiscale approach offers the possibility of space-time localization in the data analysis which would not be possible in case of a classical Fourier analysis.

\section{Acknowledgments}

The author wishes to thank Prof. Dr. Willi Freeden, chairman of the Geomathematics Group at the University of Kaiserslautern/Germany, for his support and for sharing his knowledge and his ideas.

\section{References}

[1] G. Backus: A Class of Self-Sustaining Dissipative Spherical Dynamos, Annals of Physics, 4 (1958), 372-447.

[2] G. Backus: Poloidal and Toroidal Fields in Geomagnetic Field Modelling, Review of Geophysics, 24 (1986), 75-109.

[3] G. Backus, R. Parker, E. Constable: Foundations of Geomagnetism, Cambridge University Press, Cambridge, 1996.

[4] M. Bayer: Geomagnetic Field Modelling From Satellite Data by First and Second Generation Vector Wavelets, PhD Thesis, Geomathematics Group, Department of Mathematics, University of Kaiserslautern, Shaker Verlag, Aachen, 2000.

[5] S. Beth: Multiscale Approximation by Vector Radial Basis Functions on the Sphere, PhD Thesis, Geomathematics Group, Department of Mathematics, University of Kaiserslautern, Shaker Verlag, Aachen, 2000.

[6] A. Ben-Menahem, S.J. Singh: Seismic Waves and Sources, Springer, New York, Heidelberg, Berlin, 1981.

[7] A. Charalambopoulos, G. Dassios, D.I. Fotiadis, C.V. Massalas: Note on eigenvector solutions on the Navier equation in cylindrical coordinates, Acta Mechanica, 134 (1999), 115-119. 
[8] A. Erdélyi (ed.), W. Magnus, F. Oberhettinger, F.G. Tricomi: Higher Transcendental Functions, Volume II, California Institute of Technology, Batheman Manuscript Project, McGraw-Hill Book Company, New York, St. Louis, San Francisco, Düsseldorf, Johannesburg, Kuala Lumpur, London, Mexico, Montreal, New Delhi, Panama, Rio de Janeiro, Singapore, Sydney, Toronto, 1953.

[9] W. Freeden: Multiscale Modelling of Spaceborne Geodata, B.G. Teubner Verlag, Stuttgart, Leipzig, 1999.

[10] W. Freeden, T. Gervens, M. Schreiner: Constructive Approximation on the Sphere With Applications to Geomathematics, 1st edition, Oxford University Press, Oxford, 1998.

[11] W. Freeden, V. Michel: Orthogonal Non-Bandlimited Wavelets on the Sphere, AGTMReport 235, Geomathematics Group, Department of Mathematics, University of Kaiserslautern, 2000.

[12] W. Freeden, V. Michel: Multiscale (Geo)Potential Modelling, Birkhäuser Verlag, to appear, 2002.

[13] W. Freeden, R. Reuter: A Constructive Method for Solving the Displacement Boundary-value Problem of Elastostatics by use of Global Basis Systems, Mathematical Methods in the Applied Sciences, 12 (1990), 105-128.

[14] W. Freeden, F. Schneider: Wavelet Approximation on Closed Surfaces and Their Application to Boundary-Value Problems of Potential Theory, Mathematical Methods in the Applied Sciences, 21 (1998), 129-163.

[15] W. Freeden, F. Schneider: Regularization Wavelets and Multiresolution, Inverse Problems, 14 (1998), 225-243.

[16] W. Freeden, U. Windheuser: Spherical Wavelet Transform and Its Discretization, Advances in Computational Mathematics, 5 (1996), 51-94.

[17] V.D. Kupradze: Potential Methods in the Theory of Elasticity, translated from Russian by H. Gutfreund, translation edited by I. Meroz, Israel Program for Scientific Translations, Jerusalem, 1965.

[18] W. Magnus, F. Oberhettinger, R.P. Soni: Formulas and Theorems for the Special Functions of Mathematical Physics, Springer Verlag, New York, 1966.

[19] E. Meister: Partielle Differentialgleichungen, eine Einführung für Physiker und Ingenieure in die klassische Theorie, Akademie Verlag, Berlin, 1996.

[20] V. Michel: A Wavelet Based Method for the Gravimetry Problem, in: Progess in Geodetic Science, Proceedings of the Geodetic Week 1998, edited by Willi Freeden, 283-298, Shaker Verlag, Aachen, 1998.

[21] V. Michel: A Multiscale Method for the Gravimetry Problem - Theoretical and Numerical Aspects of Harmonic and Anharmonic Modelling, PhD Thesis, Geomathematics Group, Department of Mathematics, University of Kaiserslautern, Shaker Verlag, Aachen, 1999.

[22] V. Michel: Scale Continuous, Scale Discretized and Scale Discrete Harmonic Wavelets for the Outer and the Inner Space of a Sphere and Their Application to an Inverse Problem in Geomathematics, ACHA, 12 (2002), 77-99. 
[23] J. Miklowitz: The Theory of Elastic Waves and Waveguides, North-Holland Publishing Company, Amsterdam, New York, Oxford, 1978.

[24] C. Müller: Foundations of the Mathematical Theory of Electromagnetic Waves, Springer-Verlag, Berlin, Heidelberg, New York, 1969.

[25] F. Oberhettinger: Tables of Fourier Transforms and Fourier Transforms of Distributions, Springer-Verlag, Berlin, Heidelberg, New York, London, Paris, Tokyo, Hong Kong, 1990.

[26] F. Schneider: Inverse Problems in Satellite Geodesy and Their Approximate Solution by Splines and Wavelets, PhD Thesis, Geomathematics Group, Department of Mathematics, University of Kaiserslautern, Shaker Verlag, Aachen, 1997.

[27] W.I. Smirnow: Lehrgang der Höheren Mathematik, Teil IV, VEB Deutscher Verlag der Wissenschaften, Berlin, 1979.

[28] G. Szegö: Orthogonal Polynomials, AMS Colloquium Publications, Volume XXII, Providence, Rhode Island, 1939.

[29] W. Walter: Einführung in die Theorie der Distributionen, 3rd edition, BIWissenschaftsverlag, Mannheim, Leipzig, Wien, Zürich, 1994.

[30] G.N. Watson: A Treatise on the Theory of Bessel Functions, 2nd edition, Cambridge University Press, Cambridge, 1966.

[31] E. Zeidler (ed.): Teubner-Taschenbuch der Mathematik, begründet von I.N. Bronstein und K.A. Semendjajew, Teubner Verlag, Stuttgart, Leipzig, 1996. 